\title{
Propagation Speeds of Traveling Fronts for Higher Order Autocatalytic Reaction-Diffusion Systems
}

\author{
Yuzo Hosono* \\ * Department of Information and Communication Sciences \\ Kyoto Sangyo University \\ Kyoto 603, Japan
}

Received July 21, 2006

Revised October 13, 2006

\begin{abstract}
This paper investigates the existence of traveling fronts and their propagation speeds for the two component higher order autocatalytic reaction-diffusion systems with any diffusion coefficients. Our elementary analysis of the vector fields in the phase space gives the estimate of the minimal propagation speeds in terms of the order of autocatalysis and the diffusion coefficients.
\end{abstract}

Key words: traveling fronts, propagation speed, autocatalytic reaction, phase space

\section{Introduction}

Autocatalytic reaction-diffusion systems including the Brusselator [20], the Field-Noyes model [7] and the Gray-Scott model [11], have stimulated an extensive amount of theoretical studies on waves and patterns produced by chemical reactions (see for example, [15]). One of the basic elements responsible for chemical pattern formation is traveling waves which describe the development of chemical processes. The papers by Needham et al. ([2]-[5], [16]-[19]) studied extensively the traveling waves in autocatalytic reactions. Focant and Gallay [8] and Hosono and Kawahara [14] also discussed the traveling waves for the mixed order autocatalytic two component systems and their minimal propagation speeds. The similar type of traveling waves appears in the combustion problem and the speeds of combustion waves were discussed by the several authors ([9], [23] and the references therein). Our concern is traveling fronts and their speeds for the higher order autocatalytic reaction-diffusion system of the form:

$$
\left\{\begin{array}{l}
u_{t}=d_{1} u_{x x}-k_{1} u v^{m}, \\
v_{t}=d_{2} v_{x x}+k_{2} u v^{m},
\end{array}\right.
$$

where $u$ and $v$ are concentrations of the reactant and the autocatalyst respectively, $d_{1}$ and $d_{2}$ are diffusion coefficients, and $k_{1}$ and $k_{2}$ are any positive constants.

Then, traveling front solutions for (1) are defined as follows. The nonnegative bounded functions of the form $(u(x, t), v(x, t))=(U(z), V(z))$ with $z=x-c t$ are

\footnotetext{
${ }^{*}$ This work was in part supported by Grant-in-Aid for Scientific Research No. 14540143 and No. 18540144 Japan Society for the Promotion of Science.
} 
said to be traveling front solutions for (1) when they satisfy the equations

$$
\left\{\begin{array}{l}
d_{1} U^{\prime \prime}+c U^{\prime}-k_{1} U V^{m}=0 \\
d_{2} V^{\prime \prime}+c V^{\prime}+k_{2} U V^{m}=0
\end{array}\right.
$$

with the boundary conditions

$$
P_{-} \equiv(U(-\infty), V(-\infty))=\left(0, v_{0}\right), \quad P_{+} \equiv(U(+\infty), V(+\infty))=\left(u_{0}, 0\right) .
$$

Here $u_{0}$ and $v_{0}$ are positive, and ' denotes $d / d z$. This condition is imposed so that on the far right there is only reactant and on the far left there is only autocatalyst, and these two states should be the critical states of the system (2).

By applying the comparison argument, Takase and Sleeman [22] proved that there exists the minimal wave speed $c^{*}$ such that traveling front solutions for (1) exist for each $c \geq c^{*}$ assuming that $u_{0}$ is sufficiently small for $m>1$. The purpose of this paper is to discuss the properties of the minimal wave speed $c^{*}$, especially the dependence of $c^{*}$ on the parameters $m, d_{1}$ and $d_{2}$ for arbitrarily fixed $u_{0}>0$. The method of the proofs employed here is the shooting argument which looks for the connection orbits of (2) and (3) in the 3-dim phase space. Throughout this paper, we always assume that $m>1$ without notice.

In the next section, we present the preliminary results required for the later discussions. In Section 3, we investigate the existence of traveling fronts for (1) and their minimal propagation speeds when $0<d_{1}<d_{2}$. In Section 4 , we study the same problem when $d_{1}>d_{2}>0$.

\section{The preliminary results}

We first write the equations in the dimensionless form. Introducing the dimensionless dependent variables $\tilde{u}=u / u_{0}, \tilde{v}=v /\left(r u_{0}\right)$ with $r=k_{2} / k_{1}$ and scaling the independent variables by $\tilde{t}=k_{1}\left(r u_{0}\right)^{m} t, \tilde{x}=\sqrt{k_{1}\left(r u_{0}\right)^{m}} x$, we write (1) as

$$
\left\{\begin{array}{l}
u_{t}=d_{1} u_{x x}-u v^{m} \\
v_{t}=d_{2} v_{x x}+u v^{m}
\end{array}\right.
$$

where $\sim$ is omitted for the simplicity of notations. We may also suppose that either of $d_{1}$ and $d_{2}$ is 1 by scaling the independent variable $x$. The corresponding traveling equations are

$$
\left\{\begin{array}{l}
d_{1} U^{\prime \prime}+c U^{\prime}-U V^{m}=0, \\
d_{2} V^{\prime \prime}+c V^{\prime}+U V^{m}=0,
\end{array}\right.
$$

with the boundary conditions

$$
P_{-} \equiv(U(-\infty), V(-\infty))=(0, \beta), \quad P_{+} \equiv(U(+\infty), V(+\infty))=(1,0) .
$$

Here $\beta$ is a constant to be determined. 
We already know the following properties of the traveling front solutions for (4), that is, the solutions of (5) and (6).

Proposition 1 ([3]). Assume that there exists a traveling front solution $(U(z), V(z))$ for (4). Then it satisfies the followings for all $z \in \mathbb{R}$.

(i) $0<U<1,0<V<1$.

(ii) $0<U^{\prime}<+\infty,-\infty<V^{\prime}<0$.

(iii) $U+V-1\left\{\begin{array}{l}>0, \quad \text { for } d_{2}>d_{1} \geq 0, \\ =0, \quad \text { for } d_{1}=d_{2}, \\ <0, \quad \text { for } d_{1}>d_{2} \geq 0 .\end{array}\right.$

Furthermore, $\lim _{z \rightarrow-\infty}(U(z), V(z))=(0,1)$, that is $\beta=1$, and $c>0$.

For the equal diffusion case: $d_{1}=d_{2}=1$, we know the following theorem.

Theorem 2 ([21]). Assume that $d_{1}=d_{2}=1$. Then, there exists some positive $c_{1}^{*}$ such that only for each $c \geq c_{1}^{*}$, (1) has a unique traveling front solution. Furthermore, the minimal wave speed $c_{1}^{*}$ satisfies that

$$
\frac{2}{m(m+1)} \leq c_{1}^{* 2} \leq \frac{2}{(m-1) m} .
$$

For the extreme case: $d_{1}=0$, we may assume $d_{2}=1$ without loss of generality, and have the following result.

Theorem 3 ([13]). Assume that $d_{1}=0, d_{2}=1$. Then, there exists $c_{0}^{*}$ such that only for each $c \geq c_{0}^{*}$, (1) has a unique traveling front solution. Furthermore, the minimal wave speed $c_{0}^{*}$ satisfies

$$
\frac{1}{m}<c_{0}^{* 2} \leq \frac{1}{m-1}
$$

For another extreme case: $d_{2}=0$, we may assume $d_{1}=1$ without loss of generality, and easily have the following result.

Theorem 4. Assume that $d_{1}=1, d_{2}=0$. Then, there exists a unique traveling front solution for (1) for each positive $c$.

Proof. See Appendix.

In the next two sections, on the basis of these results, we discuss the general case that the both diffusion coefficients are not zero. To do so, we require some definitions and the Wazewski theorem which was formulated by Dunbar [6]. Let $\mathbf{y}\left(z ; \mathbf{y}_{0}\right)$ be a solution of the initial value problem

$$
\mathbf{y}^{\prime}=\mathbf{f}(\mathbf{y}), \quad \mathbf{y}(0)=\mathbf{y}_{0},
$$

where $^{\prime}=d / d z, \mathbf{y}, \mathbf{y}_{0} \in \mathbb{R}^{n}$, and $\mathbf{f}(\mathbf{y})$ is Lipschitz continuous. Set $\mathbf{y}\left(z ; \mathbf{y}_{0}\right)=\mathbf{y}_{0} \cdot z$ and $V \cdot Z=\left\{\mathbf{y}_{0} \cdot z: \mathbf{y}_{0} \in V, z \in Z\right\}$. Given $W \subseteq \mathbb{R}^{n}$, let $W^{-}=\left\{\mathbf{y}_{0} \in W:\right.$ any $z>$ $\left.0, \mathbf{y}_{0} \cdot[0, z) \not \subset W\right\} . W^{-}$is called the immediate exit set. Given $\Sigma \subseteq W$, let 
$\Sigma^{0}=\left\{\mathbf{y}_{0} \in \Sigma\right.$ : there is an $z_{0}=z_{0}\left(\mathbf{y}_{0}\right)$ such that $\left.\mathbf{y}_{0} \cdot z_{0} \notin W\right\}$. For $\mathbf{y}_{0} \in \Sigma^{0}$, define $T\left(\mathbf{y}_{0}\right)=\sup \left\{z: \mathbf{y}_{0} \cdot[0, z] \subseteq W\right\}$, which is called an exit time. We denote the closure of a set $W$ by $\operatorname{cl}(W)$.

Proposition 5 ([6]). Suppose

1) If $\mathbf{y}_{0} \in \Sigma$ and $\mathbf{y}_{0} \cdot[0, z] \subseteq \operatorname{cl}(W)$, then $\mathbf{y}_{0} \cdot[0, z] \subseteq W$.

2) If $\mathbf{y}_{0} \in \Sigma, \mathbf{y}_{0} \cdot z \in W$ and $\mathbf{y}_{0} \cdot z \notin W^{-}$, then there is an open set $V_{z}$ about $\mathbf{y}_{0} \cdot z$ disjoint from $W^{-}$.

3) $\quad \Sigma=\Sigma^{0}, \Sigma$ is compact, and $\Sigma$ intersect an orbit of (9) only once in $W$.

Then the mapping $F\left(\mathbf{y}_{0}\right)=\mathbf{y}_{0} \cdot T\left(\mathbf{y}_{0}\right)$ is homeomorphism from $\Sigma$ to its image on $W^{-}$.

A set $W \subseteq \mathbb{R}^{n}$ satisfying (1) and (2) in Proposition 5 is called a Wazewski set.

\section{The case $0<d_{1}<d_{2}$}

For $d_{1} \geq 0$ and $d_{2}>0$, the system (4) can be written as

$$
\left\{\begin{array}{l}
u_{t}=d u_{x x}-u v^{m} \\
v_{t}=v_{x x}+u v^{m}
\end{array}\right.
$$

by the change of the independent variable $x$, where $d=d_{1} / d_{2}$. Then the traveling equations for (10) are

$$
\left\{\begin{array}{l}
d U^{\prime \prime}+c U^{\prime}-U V^{m}=0 \\
V^{\prime \prime}+c V^{\prime}+U V^{m}=0
\end{array}\right.
$$

The boundary conditions are specified by (6) with $\beta=1$.

Adding the above two equations and integrating the resulting equation, we have the relation $d U^{\prime}+V^{\prime}+c(U+V-1)=0$ with the aid of the boundary condition (6). Set $X=U+V-1$. Proposition 1 assures that $X$ is positive when $0<d<1$. Then (11) is reduced to the first order system

$$
\left\{\begin{array}{l}
X^{\prime}=-\frac{c}{d} X-\left(\frac{1}{d}-1\right) W \\
V^{\prime}=W \\
W^{\prime}=-c W-(1-V+X) V^{m}
\end{array}\right.
$$

The boundary conditions are

$$
\begin{aligned}
& (X(-\infty), V(-\infty), W(-\infty))=(0,1,0) \\
& (X(+\infty), V(+\infty), W(+\infty))=(0,0,0)
\end{aligned}
$$


Introducing the new dependent variables by

$$
q=V, \quad p=\frac{q^{\prime}}{q}
$$

we can write $(12)$ as

$$
\left\{\begin{array}{l}
X^{\prime}=-\frac{c}{d} X-\left(\frac{1}{d}-1\right) p q \\
q^{\prime}=p q \\
p^{\prime}=-p(p+c)-(1-q+X) q^{m-1}
\end{array}\right.
$$

For later use, we denote (15) in the vector form as $\mathbf{u}^{\prime}=\mathbf{f}_{d}(\mathbf{u})$ with $\mathbf{u}=(X, q, p)$, and $\mathbf{u}\left(z ; \mathbf{u}_{0}\right)$ denotes a solution of (15) satisfying $\mathbf{u}\left(0 ; \mathbf{u}_{0}\right)=\mathbf{u}_{0}$.

We should note that the singularity $(0,0,0)$ of $(12)$ is split into two critical points $P_{0}=(0,0,0)$ and $P_{c}=(0,0,-c)$ in $(15)$. Thus, $(15)$ has three critical points $P_{0}=(0,0,0), P_{c}=(0,0,-c)$ and $P_{1}=(0,1,0)$. The properties of these critical points are as follows. $P_{1}=(0,1,0)$ has the 1 -dim unstable manifold and the 2 -dim stable manifold. $P_{0}=(0,0,0)$ is a topologically stable node. $P_{c}=(0,0,-c)$ has the 2-dim stable manifold and the 1-dim unstable manifold.

Now our problem is to find an orbit of (15) connecting $P_{1}=(0,1,0)$ with $P_{0}=(0,0,0)$ or with $P_{c}=(0,0,-c)$, which lies entirely in $\Omega^{+}=\{(X, q, p): X>$ $0,0<q<1, p<0\}$. Here, let $\mathcal{U}_{d}(c)$ be the part of the unstable manifold of $P_{1}$ lying in $\Omega^{+}$, whose existence will be assured in Section 3.2. Our main goal of this section is to find the values of $c$ for which $\mathcal{U}_{d}(c)$ approaches $P_{0}=(0,0,0)$ or $P_{c}=(0,0,-c)$.

\subsection{The property of the vector field}

We first discuss the property of the vector field of (15) and the behavior of the unstable manifold $\mathcal{U}_{d}(c)$. To do this, we require the information on the case of $d=0$. For $d=0$, the first equation of (15) gives $X=-p q / c$ and (15) is reduced to

$$
\left\{\begin{array}{l}
q^{\prime}=p q \\
p^{\prime}=-p(p+c)-\left(1-q-\frac{p q}{c}\right) q^{m-1}
\end{array}\right.
$$

which has three critical points $(0,0),(1,0)$ and $(0,-c)$. The proof of Theorem 3 assures the followings. There exists a unique orbit of $(16)$ connecting $(1,0)$ to $(0,-c)$ if $c=c_{0}^{*}$ and to $(0,0)$ if $c>c_{0}^{*}$. This orbit can be represented by $p=\psi_{c}(q)<0$ for $0<q<1$, and it satisfies that $\psi_{c}(1)=0$ for $c \geq c_{0}^{*}, \psi_{c_{0}^{*}}(0)=c_{0}^{*}$, and $\psi_{c}(0)=0$ for $c>c_{0}^{*}$. For $c_{0}^{*}$, let $\Omega_{0}^{*}$ be the set $\left\{(q, p): 0<q<1, \psi_{c_{0}^{*}}(q)<p<0\right\}$. Then any orbit of (16) starting from a point in $\Omega_{0}^{*}$ stays in $\Omega_{0}^{*}$ for $z \geq 0$ and converges to the critical point $(0,0)$ as $z \rightarrow \infty$. Also, it holds that $\psi_{c}^{\prime}(1)=1 / c$, which is easily derived from the linearized analysis of the critical point $(1,0)$. By the use of the orbit $p=\psi_{c}(q)$, we introduce the region $\Omega_{1}$ defined by

$$
\Omega_{1} \equiv\left\{(X, q, p): 0<X<-\frac{1}{c} \psi_{c}(q) q, 0<q<1, \psi_{c}(q)<p<0\right\} .
$$


Then, the boundary of $\Omega_{1}$, denoted by $\partial \Omega_{1}$, consists of the followings:

$$
\begin{aligned}
& S_{1}=\left\{(X, q, p): 0<q<1, X=-\psi_{c}(q) \frac{q}{c}, \psi_{c}(q)<p<0\right\}, \\
& S_{2}=\left\{(X, q, p): 0<q<1,0<X<-\psi_{c}(q) \frac{q}{c}, p=\psi_{c}(q)\right\}, \\
& S_{3}=\left\{(X, q, p): 0<q<1, X=0, \psi_{c}(q) q<p<0\right\}, \\
& S_{4}=\left\{(X, q, p): 0<q<1,0<X<-\psi_{c}(q) \frac{q}{c}, p=0\right\}, \\
& J_{1}=\{(X, q, p): 0<q<1, X=0, p=0\}, \\
& J_{2}=\left\{(X, q, p): 0<q<1, X=0, p=\psi_{c}(q)\right\}, \\
& J_{3}=\left\{(X, q, p): 0<q<1, X=-\psi_{c}(q) \frac{q}{c}, p=0\right\}, \\
& J_{4}=\left\{(X, q, p): 0<q<1, X=-\psi_{c}(q) \frac{q}{c}, p=\psi_{c}(q)\right\}, \\
& I_{0}=\left\{(X, q, p): X=0, q=0, \psi_{c}(0)<p<0\right\}, \\
& P_{0}, P_{1} \text { and } P_{c} .
\end{aligned}
$$

That is,

$$
\partial \Omega_{1}=\left(\bigcup_{i=1}^{4} S_{i}\right) \cup\left(\bigcup_{i=1}^{4} J_{i}\right) \cup I_{0} \cup P_{0} \cup P_{1} \cup P_{c} .
$$

Here, note that $I_{0}=\varnothing$ for any $c>c_{0}^{*}$.

Proposition 6. Let be fixed in $(0,1)$ and $c \geq c_{0}^{*}$. Any orbit of (15) starting from a point $\mathbf{u}_{0} \in \Omega_{1}$, denoted by $\mathbf{u}\left(z ; \mathbf{u}_{0}\right)$, stays in $\Omega_{1}$ for all $z \geq 0$.

Proof. $\quad P_{0}, P_{1}$ and $P_{c}$ are critical points and $I_{0}$ is an invariant manifold, so that $\mathbf{u}\left(z ; \mathbf{u}_{0}\right)$ cannot reach any point of the set $I_{0} \cup P_{0} \cup P_{1} \cup P_{c}$ in a finite time. To prove that $\mathbf{u}\left(z ; \mathbf{u}_{0}\right)$ cannot leave $\Omega_{1}$ through $S_{i}(i=1, \ldots, 4)$, it suffices to show that the inner products of the outward normal $\mathbf{n}_{i}$ of $S_{i}$ and the vector field $\mathbf{f}_{d}$ are all negative.

We begin with $S_{1}$. On $S_{1}, \mathbf{n}_{1}=\left(1,(1 / c)\left(\psi_{c}(q)+q \psi_{c}^{\prime}(q)\right), 0\right)$. Hence we have

$$
\begin{aligned}
\mathbf{n}_{1} \cdot \mathbf{f}_{d} & =-\frac{c}{d} X-\left(\frac{1}{d}-1\right) p q+p q \frac{1}{c}\left(\psi_{c}+q \psi_{c}^{\prime}\right) \\
& =\frac{1}{d} \psi q-\frac{1}{d} p q+\frac{p q}{c}\left(c+\psi_{c}+q \psi_{c}^{\prime}\right) \leq \frac{p q}{c}\left(c+\psi_{c}+q \psi_{c}^{\prime}\right) .
\end{aligned}
$$

It follows from (16) that

$$
q \psi_{c}^{\prime}(q)=-\left(\psi_{c}+c\right)-\frac{1}{\psi_{c}}\left(1-q-\frac{\psi_{c} q}{c}\right) q^{m-1},
$$

which gives

$$
\mathbf{n}_{1} \cdot \mathbf{f}_{d} \leq-\frac{p q}{c \psi_{c}}\left(1-q-\frac{\psi_{c} q}{c}\right) q^{m-1}<0 \quad \text { for any } \quad \mathbf{u} \in S_{1} .
$$


On $S_{2}, \mathbf{n}_{2}=\left(0, \psi_{c}^{\prime}(q),-1\right)$. Hence we have

$$
\begin{aligned}
\mathbf{n}_{2} \cdot \mathbf{f}_{d}= & \psi_{c}^{\prime} p q+p(p+c)+(1-q+X) q^{m-1} \\
= & -\psi_{c}\left(\psi_{c}+c\right)-\left(1-q-\frac{\psi_{c} q}{c}\right) q^{m-1} \\
& +\psi_{c}\left(\psi_{c}+c\right)+(1-q+X) q^{m-1} \\
= & \left(X+\frac{\psi_{c} q}{c}\right) q^{m-1}<0 \text { for any } \mathbf{u} \in S_{2} .
\end{aligned}
$$

On $S_{3}, \mathbf{n}_{3}=(-1,0,0)$, so that

$$
\mathbf{n}_{3} \cdot \mathbf{f}_{d}=\frac{c}{d} X+\left(\frac{1}{d}-1\right) p q=\left(\frac{1}{d}-1\right) p q<0, \quad \text { for any } \quad \mathbf{u} \in S_{3} .
$$

Similarly, on $S_{4}, \mathbf{n}_{4}=(0,0,1)$, so that

$$
\begin{array}{r}
\mathbf{n}_{4} \cdot \mathbf{f}_{d}=-p(p+c)-(1-q+X) q^{m-1}=-(1-q+X) q^{m-1}<0, \\
\text { for any } \mathbf{u} \in S_{4} .
\end{array}
$$

For the remaining part of the boundary $J_{i}(i=1, \ldots, 4)$, we have to examine the orbit $\mathbf{u}\left(z ; \mathbf{u}_{0}\right)$ for $\mathbf{u}_{0} \in J_{i}$. For $\mathbf{u}_{0}=\left(0, q_{0}, 0\right) \in J_{1}$, we see that

$$
\begin{aligned}
\mathbf{u}\left(z ; \mathbf{u}_{0}\right)= & \mathbf{u}_{0}+\mathbf{u}^{\prime}\left(0 ; \mathbf{u}_{0}\right) z+\frac{1}{2} \mathbf{u}^{\prime \prime}\left(0 ; \mathbf{u}_{0}\right) z^{2}+(\text { h.o.t }) \\
= & \mathbf{u}_{0}+\mathbf{f}_{d}\left(\mathbf{u}_{0}\right) z+\frac{1}{2} \operatorname{Df}_{d}\left(\mathbf{u}_{0}\right) \mathbf{u}^{\prime}\left(0 ; \mathbf{u}_{0}\right) z^{2}+(\text { h.o.t }) \\
= & \left(0, q_{0}, 0\right)+\left(0,0,-\left(1-q_{0}\right) q_{0}^{m-1}\right) z \\
& \left.+\frac{1}{2}\left(1-q_{0}\right) q_{0}^{m-1}\left(\left(\frac{1}{d}-1\right) q_{0},-q_{0}, c\right) z^{2}+\text { (h.o.t }\right),
\end{aligned}
$$

where $\mathrm{Df}_{d}(\mathbf{u})$ is the Jacobi matrix of $\mathbf{f}_{d}(\mathbf{u})$. This shows that $X\left(z ; \mathbf{u}_{0}\right)>0$ and $p\left(z ; \mathbf{u}_{0}\right)<0$ for sufficiently samll positive $z$, so that any orbit $\mathbf{u}\left(z ; \mathbf{u}_{0}\right)$ for $\mathbf{u}_{0} \in \Omega_{1}$ cannot traverse $J_{1}$. Similar argument assures that the same result holds for $J_{2}, J_{3}$ and $J_{4}$. This completes the proof.

\subsection{The local property of $\mathcal{U}_{d}(c)$}

We next discuss the local behavior of $\mathcal{U}_{d}(c)$ near the critical point $P_{1}$. The linearization of (15) at $P_{1}$ becomes

$$
\left(\begin{array}{ccc}
-\frac{c}{d} & 0 & 1-\frac{1}{d} \\
0 & 0 & 1 \\
-1 & 1 & -c
\end{array}\right)
$$

Its characteristic equation becomes

$$
g(\lambda, c, d) \equiv\left(\lambda+\frac{c}{d}\right) f^{c}(\lambda)-\left(\frac{1}{d}-1\right) \lambda=0,
$$


with $f^{c}(\lambda)=\left(\lambda^{2}+c \lambda-1\right)$. This can be factorized as

$$
\begin{aligned}
g(\lambda, c, d) & =(\lambda+c)\left(\lambda^{2}+\frac{c}{d} \lambda-\frac{1}{d}\right) \\
& =(\lambda+c)\left(\lambda-\lambda_{-}^{d}(c)\right)\left(\lambda-\lambda_{+}^{d}(c)\right),
\end{aligned}
$$

where $\lambda_{-}^{d}(c)=-(1 /(2 d))\left(c+\sqrt{c^{2}+4 d}\right)<0<\lambda_{+}^{d}(c)=-(1 /(2 d))\left(c-\sqrt{c^{2}+4 d}\right)$. Therefore, (17) has two negative eigenvalues $-c$ and $\lambda_{-}^{d}(c)$, and one positive eigenvalue $\lambda_{+}^{d}(c)$. For $d=1$, we see that $\lambda_{ \pm}^{1}=(1 / 2)\left(-c \pm \sqrt{c^{2}+4}\right)$ are zeros of $f^{c}(\lambda)$. Moreover, it is obvious that $\lambda_{+}^{d}(c)$ is strictly monotone decreasing with respect to $d>0$, since $\lambda_{+}^{d}(c)=-(1 /(2 d))\left(c-\sqrt{c^{2}+4 d}\right)=2 /\left(\sqrt{c^{2}+4 d}+c\right)$. The eigenvector $\mathbf{p}_{+}^{d}(c)$ corresponding to the eigenvalue $\lambda_{+}^{d}(c)$ is given by $\mathbf{p}_{+}^{d}(c)=$ $\left(-f^{c}\left(\lambda_{+}^{d}(c)\right), 1, \lambda_{+}^{d}(c)\right)$ for $d>0$.

Thus we have the following two propositions.

Proposition 7. Let $c$ be fixed positive. For $d>0, \mathcal{U}_{d}(c)$ has the tangential direction $\mathbf{p}_{+}^{d}(c)=\left(-f^{c}\left(\lambda_{+}^{d}(c)\right), 1, \lambda_{+}^{d}(c)\right)$ at $P_{1}$, and $\lambda_{+}^{d}(c)$ and $f^{c}\left(\lambda_{+}^{d}(c)\right)$ are both strictly monotone decreasing with respect to $d$. Therefore, it holds that if $0<d<1$,

$$
0=f^{c}\left(\lambda_{+}^{1}(c)\right)<f^{c}\left(\lambda_{+}^{d}(c)\right)<f^{c}\left(\lambda_{+}^{0}(c)\right)=\frac{1}{c} \lambda_{+}^{0}(c) .
$$

and if $d>1$,

$$
-1=f^{c}(0)<f^{c}\left(\lambda_{+}^{d}(c)\right)<f^{c}\left(\lambda_{+}^{1}(c)\right)=0 .
$$

Proof. We already see that $\lambda_{+}^{d}(c)$ is strictly monotone decreasing with respect to $d$, so that the monotonicity of $f^{c}\left(\lambda_{+}^{d}\right)$ with respect to $d$ are also obvious because $f^{c^{\prime}}(\lambda)=2 \lambda+c>0$ for any $\lambda>0$. This completes the proof.

Proposition 8. Let d be fixed positive. For $c>0, \lambda_{+}^{d}(c)$ is strictly monotone decreasing with respect to $c$, and

$$
\frac{\partial}{\partial c} f^{c}\left(\lambda_{+}^{d}(c)\right) \begin{cases}<0, & \text { for } 0<d<1 \\ >0, & \text { for } d>1 .\end{cases}
$$

Proof. The expression $\lambda_{+}^{d}=-(1 /(2 d))\left(c-\sqrt{c^{2}+4 d}\right)$ directly shows that $(\partial / \partial c) \lambda_{+}^{d}=-\lambda_{+}^{d} / \sqrt{c^{2}+4 d}<0$. Since $(\partial / \partial c) f^{c}\left(\lambda_{+}^{d}(c)\right)=2(d-1)\left(2 \lambda_{+}^{d}(c)^{2}\right) / \sqrt{c^{2}+4 d}$, (21) is obvious, so that the proof is completed.

We next show that $\mathcal{U}_{d}(c)$ enters $\Omega_{1}$. Since $\mathcal{U}_{d}(c)$ has the tangential direction $\mathbf{p}_{+}^{d}$ at $P_{1}$, the projection of $\mathcal{U}_{d}(c)$ to the plane $X=0$ has a slope $\lambda_{+}^{d}$ and the projection of $\mathcal{U}_{d}(c)$ to the plane $p=0$ has a slope $-f\left(\lambda_{+}^{d}\right)$. On the other hand, the boundary surfaces $p=\psi_{c}(q)$ and $X=-\psi_{c}(q) q / c$ have slopes $\lambda_{+}^{0}=1 / c$ and $-\lambda_{+}^{0} / c$, respectively at $q=1$. Therefore, the inequalities (19) assures that $\mathcal{U}_{d}(c)$ enters $\Omega_{1}$. Thus, from Proposition 6 we can conclude that $\mathcal{U}_{d}(c)$ stays in $\Omega_{1}$ for all 
$z \in \mathbb{R}$. There exists no critical point in $\Omega_{1}$ and $q^{\prime}=p q<0$, so that $q \rightarrow 0$. This implies that $X(q) \rightarrow 0$ as $q \rightarrow 0$, and hence $\mathcal{U}_{d}(c)$ approaches $\mathrm{cl}\left(I_{0}\right)$, which consists of the ordinary points $I_{0}$ and the critical points $P_{0}$ and $P_{c}$. Therefore $\mathcal{U}_{d}(c)$ has to approach $P_{0}$ or $P_{c}$. In fact, the following lemma holds.

Lemma 9. Assume that $0<d<1$. Then, for each $c \geq c_{0}^{*}$, there exists an orbit of (15) which connects $P_{1}$ with $P_{0}$.

Proof. It suffices to show that $\mathcal{U}_{d}(c)$ cannot approach to $P_{c}$ as $z \rightarrow \infty$ for $c=c_{0}^{*}$ because $\Omega_{1}$ does not contain $P_{c}$ for any $c>c_{0}^{*}$. Assume that $c=c_{0}^{*}$ in the following. We first choose $\mathbf{u}_{0}=\left(X_{0}, q_{0}, p_{0}\right) \in \mathcal{U}_{d}$ such that $p_{0} /\left(q_{0}-1\right)<$ $\psi_{c}\left(q_{0}\right) /\left(q_{0}-1\right)$, which is possible since

$$
\lim _{q_{0} \rightarrow 1} \frac{p_{0}}{q_{0}-1}=\lambda_{+}^{d}<\lambda_{+}^{0}=\frac{1}{c}=\lim _{q_{0} \rightarrow 1} \frac{\psi_{c}\left(q_{0}\right)}{q_{0}-1} .
$$

Let $\mathbf{q}_{0}$ be $\left(q_{0}, p_{0}\right)$ and $\left(q\left(z ; \mathbf{q}_{0}\right), p\left(z ; \mathbf{q}_{0}\right)\right)$ be the solution of (16) satisfying $\left(q\left(0 ; \mathbf{q}_{0}\right), p\left(0 ; \mathbf{q}_{0}\right)\right)=\mathbf{q}_{0}$. Then, $\mathbf{q}_{0} \in \Omega_{0}^{*}$, so that $\left(q\left(z ; \mathbf{q}_{0}\right), p\left(z ; \mathbf{q}_{0}\right)\right)$ stays in $\Omega_{0}^{*}$ for all $z \geq 0$ and converges to $(0,0)$ as $z \rightarrow \infty$. We can represent this orbit by $p=\tilde{\psi}_{c}(q),\left(0 \leq q \leq q_{0}\right)$, where $\tilde{\psi}_{c}(0)=0$ and $\tilde{\psi}_{c}\left(q_{0}\right)=p_{0}$. Now we define the region $\tilde{\Omega}_{1}$ by $\left\{(X, q, p): 0<X<-(1 / c) \tilde{\psi}_{c}(q) q, 0<q<q_{0}, \tilde{\psi}_{c}(q)<p<0\right\}$. Repeating the same arguments in the proof of Proposition 6 , we conclude that $\mathcal{U}_{d}(c)$ stays in $\tilde{\Omega}_{1}$ for all $z \geq 0$ and converges to $(0,0,0)$ as $z \rightarrow \infty$ because $\tilde{\psi}_{c}(q) \rightarrow 0$ as $q \rightarrow 0$. This completes the proof.

\subsection{The existence of the orbit connecting $\boldsymbol{P}_{1}$ and $\boldsymbol{P}_{c}$}

In this subsection, we shall prove the existence of the orbit connecting $P_{1}$ and $P_{c}$ with the aid of the Wazewski theorem. In order to apply the Wazewski theorem, we rewrite the system (15) as

$$
\left\{\begin{array}{l}
X^{\prime}=-\frac{c}{d} X-\left(\frac{1}{d}-1\right) p q \\
q^{\prime}=p q \\
p^{\prime}=-p(p+c)-(1-q+X) q^{m-1} \\
c^{\prime}=0
\end{array}\right.
$$

which is simply denoted by the vector notation: $\mathbf{U}^{\prime}=\mathbf{F}(\mathbf{U})$ with $\mathbf{U}=(\mathbf{u}, c)=$ $(X, q, p, c)$, and $\mathbf{U}\left(z ; \mathbf{U}_{0}\right)$ denotes a solution of $(22)$ which satisfies $\mathbf{U}\left(0 ; \mathbf{U}_{0}\right)=\mathbf{U}_{0}$. This system has three critical manifolds $\tilde{P}_{0}(c)=(0,0,0, c), \tilde{P}_{1}(c)=(0,1,0, c)$ and $\tilde{P}_{2}(c)=(0,0,-c, c)$. In $\mathbb{R}^{4}$, we shall construct the Wazewski set for $(22)$.

Let $W_{c}$ be

$$
W_{c}=\left\{\mathbf{u}=(X, q, p): 0 \leq X \leq 1, l_{c}(p) \leq q \leq 1,-c \leq p \leq 0\right\},
$$


where $l_{c}(p)$ is defined by

$$
l_{c}(p)= \begin{cases}\left(\frac{1}{k}(p+c)\right)^{1 /(m-1)}, & -c \leq p \leq-\frac{c}{2} \\ q_{k}, & -\frac{c}{2} \leq p \leq 0\end{cases}
$$

with $q_{k}=(c /(2 k))^{1 /(m-1)}$. We also define $\tilde{W}$ by $\tilde{W}=\left\{\mathbf{U}=(\mathbf{u}, c): \mathbf{u} \in W_{c}, c_{1} \leq\right.$ $\left.c \leq c_{2}\right\}$, where $c_{1}$ and $c_{2}$ are any constants satisfying $0<c_{1}<c_{2}$ and will be specified later. The boundary of $\tilde{W}$ is consists of $\partial \tilde{W}_{1}=\left\{\mathbf{U}=(\mathbf{u}, c): \mathbf{u} \in \partial W_{c}, c_{1} \leq c \leq\right.$ $\left.c_{2}\right\}$ and $\partial \tilde{W}_{2}=\bigcup_{i=1}^{2}\left\{\mathbf{U}=(\mathbf{u}, c): \mathbf{u} \in \operatorname{int}\left(W_{c}\right), c=c_{i}\right\}$, that is, $\partial \tilde{W}=\partial \tilde{W}_{1} \cup \partial \tilde{W}_{2}$. Here, for each fixed $c$, int $\left(W_{c}\right)$ and $\partial W_{c}$ denote the interior and the boundary of $W_{c}$ in $\mathbb{R}^{3}$, respectively. $\partial W_{c}$ consists of two critical points $P_{1}$ and $P_{c}$, and the following surfaces $H_{c}^{i}(i=1,2, \ldots, 6)$ and the one dimensional invariant manifold $J_{c}$.

$$
\begin{aligned}
& H_{c}^{1}=\left\{(X, q, p): 0 \leq X \leq 1, q=l_{c}(p),-c<p \leq 0\right\} \\
& H_{c}^{2}=\{(X, q, p): 0 \leq X \leq 1,0<q \leq 1, p=-c\} \\
& H_{c}^{3}=\{(X, q, p): 0 \leq X \leq 1, q=1,-c<p<0\} \\
& H_{c}^{4}=\left\{(X, q, p): 0 \leq X \leq 1, \frac{c}{2 k}<q \leq 1, p=0\right\} \backslash P_{1}, \\
& H_{c}^{5}=\left\{(X, q, p): X=0, l_{c}(p)<q<1,-c<p<0\right\} \\
& H_{c}^{6}=\left\{(X, q, p): X=1, l_{c}(p)<q<1,-c<p<0\right\} \\
& J_{c}=\{(X, q, p): 0<X \leq 1, q=0, p=-c\} .
\end{aligned}
$$

That is, $\partial W_{c}$ is the disjoint union of these sets, and expressed as

$$
\partial W_{c}=\left(\bigcup_{i=1}^{6} H_{c}^{i}\right) \cup J_{c} \cup P_{1} \cup P_{c} .
$$

Set $\tilde{H}^{i}=\left\{(\mathbf{u}, c): \mathbf{u} \in H_{c}^{i}, c_{1} \leq c \leq c_{2}\right\}$ for $i=1,2$. Then, we have the proposition.

Proposition 10. For sufficiently large positive $k$, the immediate exit set $\tilde{W}^{-}$ of $\tilde{W}$ is the disjoint union of $\tilde{H}^{1}$ and $\tilde{H}^{2}$, that is,

$$
\tilde{W}^{-}=\tilde{H}^{1} \cup \tilde{H}^{2}, \quad \tilde{H}^{1} \cap \tilde{H}^{2}=\varnothing,
$$

for any $c_{1}$ and $c_{2}$ satisfying $0<c_{1}<c_{2}$.

Proof. It is obvious that $\partial \tilde{W}_{2}$ is not an immediate exit set because $\operatorname{int}\left(W_{c}\right)$ is open in $\mathbb{R}^{3}$ and $\left\{(\mathbf{u}, c): c=c_{i}\right\} \quad(i=1,2)$ are invariant manifolds. Therefore, it suffices to discuss $\partial \tilde{W}_{1}$. We denote the outward normal of $\partial \tilde{W}_{1}$ by $\tilde{\mathbf{n}}$. 
Let us first consider $\tilde{H}^{1}$. For $-c<p \leq-c / 2, q=l_{c}(p)$, which implies $p=k q^{m-1}-c\left(0<q \leq q_{k}\right)$. This gives $\tilde{\mathbf{n}}=\left(0,-k(m-1) q^{m-2}, 1,1\right)$ on $\tilde{H}^{1}$ for $-c<p \leq-c / 2$. Thus, noting that $0 \leq X \leq 1$ in $\operatorname{cl}(\tilde{W})$, we have

$$
\begin{aligned}
\tilde{\mathbf{n}} \cdot \mathbf{F} & =-k(m-1) p q^{m-1}-p(p+c)-(1-q+X) q^{m-1} \\
& =-k(m-1) p q^{m-1}-k p q^{m-1}-(1-q+X) q^{m-1} \\
& =-m k q^{m-1}\left(p+\frac{1}{m k}(1-q+X)\right) \\
& \geq m k q^{m-1}\left(\frac{c}{2}-\frac{2}{m k}\right)>0,
\end{aligned}
$$

if we choose $k>4 /\left(m c_{1}\right)$. For $-c / 2<p<0, q=q_{k}>0$, so that on $H_{c}^{1}$ we see that $\tilde{\mathbf{n}} \cdot \mathbf{F}=-p q_{k}>0$. On $E_{1}=\left\{(X, q, p, c): 0 \leq X \leq 1, q=q_{k}, p=\right.$ $\left.0, c_{1} \leq c \leq c_{2}\right\}$, we have $\tilde{\mathbf{U}}^{\prime}=\tilde{\mathbf{F}}=\left(-(c / d) X, 0,-\left(1-q_{k}+X\right) q_{k}^{m-1}, 0\right)$. Hence for $\tilde{\mathbf{U}}_{0}=\left(X_{0}, q_{k}, 0, c_{0}\right) \in E_{1}$

$$
\begin{aligned}
q\left(z: \tilde{\mathbf{U}}_{0}\right) & =q\left(0: \tilde{\mathbf{U}}_{0}\right)+q^{\prime}\left(0: \tilde{\mathbf{U}}_{0}\right) z+\frac{1}{2} q^{\prime \prime}\left(0: \tilde{\mathbf{U}}_{0}\right) z^{2}+O\left(z^{3}\right) \\
& =q_{k}+\frac{1}{2} p^{\prime}\left(0: \tilde{\mathbf{U}}_{0}\right) q\left(0: \tilde{\mathbf{U}}_{0}\right) z^{2}+O\left(z^{3}\right) \\
& =q_{k}-\frac{1}{2}\left(1-q_{k}+X_{0}\right) q_{k}^{m} z^{2}+O\left(z^{3}\right)<q_{k}
\end{aligned}
$$

for any sufficiently small positive $z$, since we can choose $k$ so large as $q_{k}=$ $\left(c_{0} /(2 k)\right)^{1 /(m-1)}<1 / 2$. Therefore, we know that $\tilde{H}^{1}$ is the immediate exit set.

Next, let us consider $\tilde{H}^{2}$. Since $\tilde{\mathbf{n}}=(0,0,-1,-1)$ on $\tilde{H}^{2}$, we see that

$$
\tilde{\mathbf{n}} \cdot \mathbf{F}=p(p+c)+(1-q+X) q^{m-1}=(1-q+X) q^{m-1}>0
$$

for $q \neq 1$ or $X \neq 0$. On $E_{2}=\left\{(X, q, p, c): X=0, q=1, p=-c, c_{1} \leq c \leq c_{2}\right\}$, $\tilde{\mathbf{F}}=(((1 / d)-1) c,-c, 0,0)$, so that

$$
\begin{aligned}
p^{\prime \prime} & =-(2 p+c) p^{\prime}-(m-1)(1+X) q^{m-2} q^{\prime}+m q^{m-1} q^{\prime}-X^{\prime} q^{m-1} \\
& =-(m-1) q^{\prime}+m q^{\prime}-X^{\prime}=q^{\prime}-X^{\prime}=-c-\left(\frac{1}{d}-1\right) c=-\frac{c}{d}<0 .
\end{aligned}
$$

Hence, for $\tilde{\mathbf{U}}_{0}=(0,1,-c, c) \in E_{2}$ we have

$$
\begin{aligned}
p\left(z: \tilde{\mathbf{U}}_{0}\right) & =p\left(0: \tilde{\mathbf{U}}_{0}\right)+p^{\prime}\left(0: \tilde{\mathbf{U}}_{0}\right) z+\frac{1}{2} p^{\prime \prime}\left(0: \tilde{\mathbf{U}}_{0}\right) z^{2}+O\left(z^{3}\right) \\
& =-c-\frac{c}{2 d} z^{2}+O\left(z^{3}\right)<-c
\end{aligned}
$$

for any sufficiently small positive $z$. Thus we also know that $\tilde{H}^{2}$ is the immediate exit set. The disjointness of $\tilde{H}^{1}$ and $\tilde{H}^{2}$ is obvious. 
Similarly, we can show that any orbit starting from

$$
\tilde{W}^{+}=\left\{\mathbf{U}=(\mathbf{u}, c): \mathbf{u} \in \bigcup_{i=3}^{6} H_{c}^{i}, c_{1} \leq c \leq c_{2}\right\},
$$

enters immediately into int $(\tilde{W})$ for any $c_{1}$ and $c_{2}$ satisfying $0<c_{1}<c_{2}$, which completes the proof.

Now, we shall prove the following lemma.

Lemma 11. Assume that $0<d<1$. Then, there exists some positive constant $c_{d}^{*}\left(<c_{0}^{*}\right)$ such that, for $c=c_{d}^{*}$, (15) has an orbit connecting $P_{1}$ with $P_{c}$.

Proof. We first consider the case that $m \geq 2$, which assures that $\mathbf{F}(\mathbf{U})$ of the system (22) is Lipschitz continuous. In order to apply the Wazewski theorem, we first identify $\Sigma$ as follows. Let $\mathbf{u}_{c}=\left(X_{c}, q_{c}, p_{c}\right)$ be a point on the unstable manifold $\mathcal{U}_{d}(c)$ for each $c$ satisfying $c_{1} \leq c \leq c_{2}$, where $q_{c}$ is chosen sufficiently close to 1 and satisfies $0<q_{c}<1$. Set $\Sigma=\left\{\mathbf{U}_{c}=\left(\mathbf{u}_{c}, c\right): c_{1}+\epsilon \leq c \leq c_{2}-\epsilon\right\} \subset \tilde{W}$ with sufficiently small $\epsilon>0$. Then $\Sigma$ is compact and intersects a trajectory of (22) only onces. $\tilde{W}$ is seen to be a Wazewski set. In fact, (1) in Proposition 5 is trivially satisfied since $\tilde{W}$ is closed. If $\mathbf{U}_{c} \in \Sigma, \mathbf{U}_{c} \cdot z \in \tilde{W}$ and $\mathbf{U}_{c} \cdot z \notin \tilde{W}^{-}$, then $\mathbf{U}_{c} \cdot z \in \operatorname{int}(\tilde{W}) \cup \partial \tilde{W}_{2} \cup\left(\partial \tilde{W}_{1} \backslash \tilde{W}^{-}\right)$. The definition of $\Sigma$ assures that $\mathbf{U}_{c} \cdot z \notin \partial \tilde{W}_{2}$. Here we note that $\partial \tilde{W}_{1} \backslash \tilde{W}^{-}=\tilde{W}^{+} \cup \tilde{W}^{0}$ with $\tilde{W}^{0}=\{(\mathbf{u}, c): \mathbf{u} \in$ $\left.\left(J_{c} \cup P_{1} \cup P_{c}\right), c_{1} \leq c \leq c_{2}\right\}$. In the proof of Proposition 10 we already see that the direction of the vector field is inward on $\tilde{W}^{+}$, so that $\mathbf{U}_{c} \cdot z \notin \tilde{W}^{+}$. Since $\tilde{W}^{0}$ consists of the invariant manifold and the critical points, it is also obvious that $\mathbf{U}_{c} \cdot z \notin \tilde{W}^{0}$. Therefore, it holds that $\mathbf{U}_{c} \cdot z \in \operatorname{int}(\tilde{W})$, which assures (2).

Now, we consider the behavior of $\mathcal{U}_{d}(c)$ for large $c$ and for small $c>0$. For any $c \geq c_{0}^{*}$, Lemma 9 asserts that $\mathcal{U}_{d}(c)$ approaches to $P_{0}$ as $z \rightarrow \infty$. This assures that $\mathcal{U}_{d}(c)$ has to leave $W_{c}$ through $H_{c}^{1}$ for $c \geq c_{0}^{*}$. Of course, it cannot traverse $H_{c}^{2}$ before it hits $H_{c}^{1}$ because $H_{c}^{1}$ is the immediate exit set of $W_{c}$. Thus we see that any orbit starting from $\mathbf{U}_{c} \in \Sigma$ hits $\tilde{H}^{1}$ with an exit time $T\left(\mathbf{U}_{c}\right)$ for $c \geq c_{0}^{*}$. Since the slope of the projection of $\mathcal{U}_{d}(c)$ to the $p$ - $q$ plane at $P_{1}$ is $\lambda_{+}^{d}(c)$ which approaches to $1 / \sqrt{d}$ as $c \rightarrow 0$, there exists some small positive $c_{1}{ }^{\prime}$ such that $\mathcal{U}_{d}(c)$ intersects the plane $p=-c$ for $c \in\left(0, c_{1}{ }^{\prime}\right]$, which implies that $\mathcal{U}_{d}(c)$ goes out from $W_{c}$ through $H_{c}^{2}$. Hence we see that an orbit starting from $\mathbf{U}_{c} \in \Sigma$ hits $\tilde{H}^{2}$ with an exit time $T\left(\mathbf{U}_{c}\right)$ for any $c \in\left(0, c_{1}{ }^{\prime}\right]$. Choose $c_{1}+\epsilon=c_{1}{ }^{\prime}$ and $c_{2}-\epsilon=c_{0}^{*}$. Then, if $\Sigma=\Sigma^{0}$, Proposition 5 says that $F(\Sigma)$ is the continuous image of the connected set $\Sigma$ on $\tilde{W}^{-}=\tilde{H}^{1} \cup \tilde{H}^{2}$. On the other hand, we have shown in the above that $F\left(\mathbf{U}_{c}\right) \equiv \mathbf{U}_{c} \cdot T\left(\mathbf{U}_{c}\right) \in \tilde{H}^{1}$ for $c=c_{1}+\epsilon, F\left(\mathbf{U}_{c}\right) \in \tilde{H}^{2}$ for $c=c_{2}-\epsilon$ and $\tilde{H}^{1} \cap \tilde{H}^{2} \neq \varnothing$, which contradicts the connectedness of $F(\Sigma)$. Therefore, $\Sigma \neq \Sigma^{0}$, so that there exists a point $\mathbf{U}_{c^{*}}=\left(\mathbf{u}_{c^{*}}, c^{*}\right) \in \Sigma$ such that the solution $\mathbf{U}\left(z ; \mathbf{U}_{c^{*}}\right)=\left(\mathbf{u}\left(z ; \mathbf{u}_{c^{*}}\right), c^{*}\right)$ of (22) stays in $\operatorname{int}(\tilde{W})$ for all $z>0$.

Next, we show that $\mathbf{u}\left(z ; \mathbf{u}_{c^{*}}\right)$ gives an orbit connecting $P_{1}$ with $P_{c}$. Note that $\mathbf{u}\left(z ; \mathbf{u}_{c^{*}}\right)$ stays in $\operatorname{int}\left(W_{c^{*}}\right)$ for all $z>0$ and there is no critical point in 
$\operatorname{int}\left(W_{c^{*}}\right)$. In $\operatorname{int}\left(W_{c^{*}}\right), q^{\prime}=p q<0$ so that $q\left(z ; \mathbf{u}_{c^{*}}\right) \rightarrow 0$ as $z \rightarrow \infty$. This assures that $p\left(z ; \mathbf{u}_{c^{*}}\right) \rightarrow-c^{*}$ as $z \rightarrow \infty$ since $0 \leq l_{c^{*}}(p)<q \operatorname{in} \operatorname{int}\left(W_{c^{*}}\right)$ and $l_{c^{*}}(p)=0$ if and only if $p=-c^{*}$. We choose now sufficiently large positive $z_{1}$ such that $-c^{*} \leq p\left(z ; \mathbf{u}_{c^{*}}\right) \leq-c^{*} / 2$ for all $z \geq z_{1}$. It easily follows from the second equation of $(22)$ that $q\left(z ; \mathbf{u}_{c^{*}}\right) \leq q\left(z_{1} ; \mathbf{u}_{c^{*}}\right) e^{-\left(c^{*} / 2\right)\left(z-z_{1}\right)}$. The first equation of $(22)$ is integrated as

$$
X(z)=e^{-\left(c^{*} / d\right)\left(z-z_{1}\right)}\left(X\left(z_{1}\right)-\left(\frac{1}{d}-1\right) \int_{z_{1}}^{z} e^{\left(c^{*} / d\right)\left(\zeta-z_{1}\right)} p(\zeta) q(\zeta) d \zeta\right)
$$

where we omit the dependency of the initial value $\mathbf{u}_{c^{*}}$ for simplicity. By using the above estimates of $p$ and $q$, we have the following inequality.

$$
\begin{aligned}
X(z) & \leq e^{-\left(c^{*} / d\right)\left(z-z_{1}\right)}\left(X\left(z_{1}\right)+\left(\frac{1}{d}-1\right) c^{*} q\left(z_{1}\right) \int_{z_{1}}^{z} e^{\left(c^{*} / d\right)\left(\zeta-z_{1}\right)} e^{-\left(c^{*} / 2\right)\left(\zeta-z_{1}\right)} d \zeta\right) \\
& \leq e^{-\left(c^{*} / d\right)\left(z-z_{1}\right)}\left(X\left(z_{1}\right)+\left(\frac{1}{d}-1\right) c^{*} q\left(z_{1}\right) \int_{z_{1}}^{z} e^{(1 / d-1 / 2) c^{*}\left(\zeta-z_{1}\right)} d \zeta\right) \\
& \leq e^{-\left(c^{*} / d\right)\left(z-z_{1}\right)}\left(X\left(z_{1}\right)+\frac{2(1-d)}{2-d} q\left(z_{1}\right)\left[e^{(1 / d-1 / 2) c^{*}\left(z-z_{1}\right)}-1\right]\right) \\
& \leq e^{-\left(c^{*} / d\right)\left(z-z_{1}\right)} X\left(z_{1}\right)+e^{-(1 / 2) c^{*}\left(z-z_{1}\right)} q\left(z_{1}\right),
\end{aligned}
$$

which asserts that $X(z) \rightarrow 0$ as $z \rightarrow \infty$. Thus we conclude that $\lim _{z \rightarrow \infty} \mathbf{u}\left(z ; \mathbf{u}_{c^{*}}\right)=$ $P_{c^{*}}$. It is obvious that $\lim _{z \rightarrow-\infty} \mathbf{u}\left(z ; \mathbf{u}_{c^{*}}\right)=P_{1}$ since $\mathbf{u}_{c^{*}} \in \mathcal{U}_{d}\left(c^{*}\right)$.

For the case that $1<m<2$, in order to assure the Lipschitz continuity of $\mathbf{F}(\mathbf{U})$, we introduce another change of the dependent variables by

$$
\hat{q}=V^{m-1}, \quad \hat{p}=\frac{\hat{q}^{\prime}}{\hat{q}},
$$

which rewrite the system (12) as follows.

$$
\left\{\begin{array}{l}
X^{\prime}=-\frac{c}{d} X-\frac{1}{m-1}\left(\frac{1}{d}-1\right) \hat{p} \hat{q}^{1 /(m-1)} \\
\hat{q}^{\prime}=\hat{p} \hat{q}, \\
\hat{p}^{\prime}=-\frac{1}{m-1} \hat{p}(\hat{p}+(m-1) c)-(m-1)\left(1-\hat{q}^{1 /(m-1)}+X\right) \hat{q} .
\end{array}\right.
$$

Here, the critical points of this system are $\hat{P}_{0}=(0,0,0), \hat{P}_{1}=(0,1,0)$, and $\hat{P}_{0}=$ $(0,0,-(m-1) c)$. We write $(25)$ in the vector form as $\hat{\mathbf{u}}^{\prime}=\hat{\mathbf{f}}_{d}(\hat{\mathbf{u}})$ with $\hat{\mathbf{u}}=(X, \hat{q}, \hat{p})$, and $\hat{\mathbf{u}}\left(z ; \hat{\mathbf{u}}_{0}\right)$ denotes a solution of $(25)$ satisfying $\hat{\mathbf{u}}\left(0 ; \hat{\mathbf{u}}_{0}\right)=\hat{\mathbf{u}}_{0}$. Now $\hat{\mathbf{f}}_{d}(\hat{\mathbf{u}})$ is Lipshitz continuous. It follows from (14) and (24) that

$$
q=\hat{q}^{1 /(m-1)}, \quad p=\frac{1}{m-1} \hat{p} .
$$

This relation assures the one to one correspondence between orbits of (15) in the phase space $\Omega^{+}$and orbits of $(25)$ in $\hat{\Omega}^{+}=\{(X, \hat{q}, \hat{p}): X>0,0<\hat{q}<1, \hat{p}<0\}$. 
Through this correspondence, the whole of the above arguments for $m \geq 2$ is valid also for $0<m<1$ since Propositions 6, 7, 8, 10 and Lemma 9 hold for $m>1$, and hence we know the existence of some $c^{*}$ such that for $c=c^{*}(25)$ has a solution $\hat{\mathbf{u}}\left(z ; \hat{\mathbf{u}}_{0}\right)$ satisfying $\lim _{z \rightarrow-\infty} \hat{\mathbf{u}}\left(z ; \hat{\mathbf{u}}_{0}\right)=\hat{P}_{1}$ and $\lim _{z \rightarrow \infty} \hat{\mathbf{u}}\left(z ; \hat{\mathbf{u}}_{0}\right)=\hat{P}_{c^{*}}$. Again, through the correspondence (26), for this $c^{*}$ we have obtained the orbit of (12) connecting $P_{1}$ with $P_{c^{*}}$. Choosing $c_{d}^{*}=c^{*}$, we complete the proof.

REMARK 1. It follows from the proof of Lemma 11 that the traveling front solution of (11) obtained above, denoted by $\left(U_{d}\left(z ; c^{*}\right), V_{d}\left(z ; c^{*}\right)\right)$, decays to $(1,0)$ exponentially as $z \rightarrow \infty$.

Thus, we have established the existence of the traveling front solution of (11) and (6) for $c \geq c_{0}^{*}$ and $c=c_{d}^{*}$. In the next subsection, we shall prove that $c_{d}^{*}$ is the minimal wave speed of traveling front solutions and state the main theorem.

\subsection{The properties of the connection orbit}

We first discuss the existence of connection orbits of (15) for any $c>c_{d}^{*}$. We can express the orbit of (15) obtained in Lemma 11 as $(X, q, p)=\left(X^{*}(q), q, p^{*}(q)\right)$ $(0 \leq q \leq 1)$ in the phase space, since $q^{\prime}=p q<0$ in $\Omega^{+}$. Here we note that $p^{*}(0)=-c_{d}^{*}$,

$$
p^{* \prime}(q)=-\frac{p^{*}\left(p^{*}+c_{d}^{*}\right)+\left(1-q+X^{*}\right) q^{m-1}}{p^{*} q},
$$

and

$$
X^{* \prime}(q)=-\frac{c_{d}^{*}}{d} \frac{X^{*}}{p^{*} q}-\left(\frac{1}{d}-1\right),
$$

By the use of this orbit, the region $\Omega^{*}$ is defined, similarly as $\Omega_{1}$, by

$$
\Omega^{*} \equiv\left\{(X, q, p): 0<X<X^{*}(q), 0<q<1, p^{*}(q)<p<0\right\} .
$$

The boundary of $\Omega^{*}$ is given by

$$
\partial \Omega^{*}=\left(\bigcup_{i=1}^{4} S_{i}^{*}\right) \cup\left(\bigcup_{i=1}^{4} J_{i}^{*}\right) \cup I_{0}^{*} \cup P_{0} \cup P_{1} \cup P_{c_{d}^{*}}
$$

where

$$
\begin{aligned}
& S_{1}^{*}=\left\{(X, q, p): 0<q<1, X=X^{*}(q), p^{*}(q)<p<0\right\}, \\
& S_{2}^{*}=\left\{(X, q, p): 0<q<1,0<X<X^{*}(q), p=p^{*}(q)\right\}, \\
& S_{3}^{*}=\left\{(X, q, p): 0<q<1, X=0, p^{*}(q)<p<0\right\}, \\
& S_{4}^{*}=\left\{(X, q, p): 0<q<1,0<X<X^{*}(q), p=0\right\}, \\
& J_{1}^{*}=\{(X, q, p): 0<q<1, X=0, p=0\},
\end{aligned}
$$




$$
\begin{aligned}
& J_{2}^{*}=\left\{(X, q, p): 0<q<1, X=0, p=p^{*}(q)\right\}, \\
& J_{3}^{*}=\left\{(X, q, p): 0<q<1, X=X^{*}(q), p=0\right\}, \\
& J_{4}^{*}=\left\{(X, q, p): 0<q<1, X=X^{*}(q), p=p^{*}(q)\right\}, \\
& I_{0}^{*}=\left\{(X, q, p): X=0, q=0,-c_{d}^{*}<p<0\right\} .
\end{aligned}
$$

Proposition 12. Let $d$ be fixed in $(0,1)$. For each $c>c_{d}^{*}$, any orbit of (15) starting from a point $\mathbf{u}_{0} \in \Omega^{*}$, denoted by $\mathbf{u}_{c}\left(z ; \mathbf{u}_{0}\right)$, stays in $\Omega^{*}$ for all $z \geq 0$.

Proof. The proof of Proposition 6 can be applied to this proposition with the minor changes by noting that $c>c_{d}^{*}$. In fact, on $S_{1}^{*}, X=X^{*}(q)$, so that $\mathbf{n}_{1}^{*}=$ $\left(1,-X^{* \prime}, 0\right)$. Here, $\mathbf{n}_{i}^{*}$ are the outward normal of the surfaces $S_{i}^{*}(i=1,2,3,4)$. Hence, using (28) we have

$$
\begin{aligned}
\mathbf{n}_{1}^{*} \cdot \mathbf{f}_{d} & =-\frac{c}{d} X-\left(\frac{1}{d}-1\right) p q-X^{* \prime} p q \\
& =-\frac{c}{d} X^{*}-\left(\frac{1}{d}-1\right) p q+\left\{\frac{c_{d}^{*}}{d} \frac{X^{*}}{p^{*} q}+\left(\frac{1}{d}-1\right)\right\} p q \\
& =\frac{1}{d}\left(\frac{c_{d}^{*} p}{p^{*}}-c\right) X^{*}<\frac{1}{d}\left(c_{d}^{*}-c\right) X^{*}<0,
\end{aligned}
$$

for any $\mathbf{u}=(X, q, p) \in S_{1}^{*}$.

On $S_{2}^{*}, p=p^{*}(q)$, so that $\mathbf{n}_{2}^{*}=\left(0, p^{* \prime}(q),-1\right)$. We then have

$$
\begin{aligned}
\mathbf{n}_{2}^{*} \cdot \mathbf{f}_{d}= & p^{* \prime} p q+p(p+c)+(1-q+X) q^{m-1} \\
= & -p^{*}\left(p^{*}+c_{d}^{*}\right)-\left(1-q+X^{*}\right) q^{m-1} \\
& +p^{*}\left(p^{*}+c\right)+(1-q+X) q^{m-1} \\
= & p^{*}\left(c-c_{d}^{*}\right)+\left(X-X^{*}\right) q^{m-1}<0,
\end{aligned}
$$

for any $\mathbf{u} \in S_{2}^{*}$. The remaining part of the proof is the same as that of Proposition 6 . This completes the proof.

Lemma 13. Let $d$ be fixed. Then, for each $c>c_{d}^{*}$, there exists an orbit of (15) connecting $P_{1}$ with $P_{0}$ lying in $\Omega^{+}$. For each positive $c<c_{d}^{*}$, there exists no orbit of (15) connecting $P_{1}$ with $P_{0}$ or $P_{c}$.

Proof. It follows from Proposition 8 that for each $c>c_{d}^{*}, \mathcal{U}_{d}(c)$ enters $\Omega^{*}$. Then Proposition 12 assures that the orbit corresponding to $\mathcal{U}_{d}(c)$ cannot leave $\Omega^{*}$. Hence, it must approach to $P_{0}$ as $z \rightarrow \infty$ since $q^{\prime}=p q<0$ in $\Omega^{*}$ and the critical point $P_{c}$ does not belong to $\operatorname{cl}\left(\Omega^{*}\right)$, which proves the first half of this lemma.

Assume that for some $c^{*}<c_{d}^{*}$, there exists an orbit connecting $P_{1}$ with $P_{c}$ for $c=c^{*}$. Then Proposition 8 asserts the existence of an orbit connecting $P_{1}$ with $P_{0}$ for $c=c_{d}^{*}$ because of $c_{d}^{*}>c^{*}$. This contradicts the uniqueness of the orbit corresponding to $\mathcal{U}_{d}(c)$. Next, assume that for some $c^{*}<c_{d}^{*}$, there exists an orbit connecting $P_{1}$ with $P_{0}$ for $c=c^{*}$. Then, we can choose $c_{2}-\epsilon=c^{*}$ in place of 
$c_{0}^{*}$ in the proof of Lemma 11, so that from Lemma 11, we see the existence of an orbit connecting $P_{1}$ with $P_{c}$ for $c=c_{d}^{* \prime}\left(<c^{*}<c_{d}^{*}\right)$. Thus, the first part of this lemma again asserts that the existence of an orbit connecting $P_{1}$ with $P_{0}$ for $c=c_{d}^{*}$, which leads us to the contradiction. This proves the last half of the assertion, which completes the proof.

Lemmas 11 and 13 allow us to call $c_{d}^{*}$ the minimal wave speed of traveling front solutions.

REMARK 2. It follows from the proof of Lemma 13 that $c_{d}^{*}$ is determined uniquely. Also, for each $c \geq c_{d}^{*}$, the traveling front solution of (11) exists uniquely except translation since $\mathcal{U}_{d}(c)$ is the unique orbit entering $P_{1}$ as $z \rightarrow-\infty$.

Next, we consider the $d$-dependence of connection orbits.

Lemma 14. Let $d \in(0,1)$ be fixed. Then, for each $\bar{d} \in(d, 1)$, there exists an orbit of (15) connecting $P_{1}$ with $P_{0}$ in $\Omega^{+}$.

Proof. We first show that for each fixed $c>0, \mathcal{U}_{d}(c)$ lies strictly below the surface $S_{0}=\{(X, q, p): 0<q<1, X=-p q / c, p<0\}$ for any $d \in(0,1)$. The normal vector $\mathbf{n}_{0}$ of $S_{0}$ is $(c, p, q)$, so that

$$
\begin{aligned}
\mathbf{n}_{0} \cdot \mathbf{f}_{d} & =c\left\{-\frac{c}{d} X-\left(\frac{1}{d}-1\right) p q\right\}+p^{2} q+q\left\{-p(p+c)-(1-q+X) q^{m-1}\right\} \\
& =-(1-q+X) q^{m}<0,
\end{aligned}
$$

for any $\mathbf{u}=(X, q, p) \in S_{0}$. This implies that any orbit lying in $\Omega^{+}$cannot traverse $S_{0}$ from the region $X<-p q / c$ to $X>-p q / c$. On the other hand, $\mathcal{U}_{d}(c)$ is represented near $P_{1}$ by

$$
\left.(X(q), q, p(q))=(0,1,0)-\left(-f^{c}\left(\lambda_{+}^{d}(c)\right), 1, \lambda_{+}^{d}(c)\right) h+o(h)\right)
$$

for sufficiently small positive $h$. Therefore,

$$
\begin{aligned}
X(q)+p(q) \frac{q}{c} & =f^{c}\left(\lambda_{+}^{d}(c)\right) h-(1-h) \lambda_{+}^{d}(c) \frac{h}{c}+o(h) \\
& =\left(f^{c}\left(\lambda_{+}^{d}(c)\right)-\frac{\lambda_{+}^{d}(c)}{c}\right) h+o(h)<0,
\end{aligned}
$$

since Proposition 7 assures that $f^{c}\left(\lambda_{+}^{d}(c)\right) / \lambda_{+}^{d}(c)<f^{c}\left(\lambda_{+}^{0}(c)\right) / \lambda_{+}^{0}(c)=1 / c$. Thus we know that $\mathcal{U}_{d}(c)$ lies strictly below $S_{0}$ near $P_{1}$ and hence cannot traverse $S_{0}$ in $\Omega^{+}$.

We again consider the region $\Omega^{*}$ and prove that $\mathcal{U}_{\bar{d}}\left(c_{d}^{*}\right)$ stays in $\Omega^{*}$ for all $z$ for $\bar{d} \in(d, 1)$. For simplicity, we denote $c_{d}^{*}$ by $c^{*}$ in the following. Proposition 7 
assures that $\mathcal{U}_{\bar{d}}\left(c^{*}\right)$ enters $\Omega^{*}$ for $\bar{d}>d$. On the surface $S_{1}^{*}$, using (28) with $c_{d}^{*}=c^{*}$, we have

$$
\begin{aligned}
\mathbf{n}_{1}^{*} \cdot \mathbf{f}_{\bar{d}} & =-\frac{c^{*}}{\bar{d}} X-\left(\frac{1}{\bar{d}}-1\right) p q-X^{* \prime} p q \\
& =-\frac{c^{*}}{\bar{d}} X^{*}-\left(\frac{1}{\bar{d}}-1\right) p q+\left\{\frac{c^{*}}{d} \frac{X^{*}}{p^{*} q}+\left(\frac{1}{d}-1\right)\right\} p q \\
& =-c^{*}\left(\frac{1}{\bar{d}}-\frac{1}{d} \frac{p}{p^{*}}\right) X^{*}-\left(\frac{1}{\bar{d}}-\frac{1}{d}\right) p q \\
& \leq-\left(\frac{1}{\bar{d}}-\frac{1}{d}\right)\left(c^{*} X^{*}+p q\right)<0,
\end{aligned}
$$

for any $\mathbf{u}=(X, q, p) \in S_{1}^{*}$, since $\mathcal{U}_{d}\left(c^{*}\right)$ stays in the region below $S_{0}$ where $X^{*}<$ $-p q / c^{*}$.

On $S_{2}^{*}, p=p^{*}(q)$, so that $\mathbf{n}_{2}^{*}=\left(0, p^{* \prime}(q),-1\right)$. We then have

$$
\begin{aligned}
\mathbf{n}_{2}^{*} \cdot \mathbf{f}_{d}= & p^{* \prime} p q+p\left(p+c^{*}\right)+(1-q+X) q^{m-1} \\
= & -p^{*}\left(p^{*}+c^{*}\right)-\left(1-q+X^{*}\right) q^{m-1} \\
& +p^{*}\left(p^{*}+c^{*}\right)+(1-q+X) q^{m-1} \\
= & \left(X-X^{*}\right) q^{m-1}<0
\end{aligned}
$$

for any $\mathbf{u} \in S_{2}^{*}$. By repeating the same argument as in Proposition 12, we can conclude that $\mathcal{U}_{\bar{d}}\left(c^{*}\right)$ stays in $\Omega^{*}$ for all $z$ for $\bar{d} \in(d, 1)$.

Next, assume that $\mathcal{U}_{\bar{d}}\left(c^{*}\right)$ connects $P_{1}$ with $P_{c^{*}}$. We can express this orbit by $\left(X_{\bar{d}}(q), q, p_{\bar{d}}(q)\right)(0 \leq q \leq 1)$. Since $\mathcal{U}_{\bar{d}}\left(c^{*}\right)$ stays in $\Omega^{*}$ for $0<q<1$, it holds that $0<X_{\bar{d}}(q)<X^{*}(q)$ and $p^{*}(q)<p_{\bar{d}}(q)<0$ for $0<q<1$. We should note that

$$
\begin{gathered}
X_{\bar{d}}(0)=X^{*}(0)=X_{\bar{d}}(1)=X^{*}(1)=0, \\
p_{\bar{d}}(0)=p^{*}(0)=-c^{*}, \quad p_{\bar{d}}(1)=p^{*}(1)=0,
\end{gathered}
$$

and

$$
\begin{gathered}
X_{\bar{d}}^{\prime}(1)=-f^{c^{*}}\left(\lambda_{+}^{\bar{d}}\left(c^{*}\right)\right), \quad X^{*^{\prime}}(1)=-f^{c^{*}}\left(\lambda_{+}^{d}\left(c^{*}\right)\right), \\
p_{\bar{d}}{ }^{\prime}(1)=\lambda_{+}^{\bar{d}}\left(c^{*}\right), \quad p^{* \prime}(1)=\lambda_{+}^{d}\left(c^{*}\right) .
\end{gathered}
$$

Since $p^{*}(q)$ and $p_{d}(q)$ satisfy

$$
\frac{d}{d q} p^{*}=-\frac{p^{*}\left(p^{*}+c^{*}\right)+\left(1-q+X^{*}\right) q^{m-1}}{p^{*} q},
$$

and

$$
\frac{d}{d q} p_{\bar{d}}=-\frac{p_{\bar{d}}\left(p_{\bar{d}}+c^{*}\right)+\left(1-q+X_{d}\right) q^{m-1}}{p_{\bar{d}} q},
$$


respectively, we have

$$
\begin{aligned}
\frac{d}{d q}\left(p^{*}-p_{\bar{d}}\right) & =-\frac{1}{q}\left(p^{*}-p_{\bar{d}}\right)-\left(\frac{1-q+X^{*}}{p^{*}}-\frac{1-q+X_{\bar{d}}}{p_{\bar{d}}}\right) q^{m-2} \\
& =-\frac{a(q)}{q}\left(p_{\bar{d}}-p^{*}\right)+b(q),
\end{aligned}
$$

where $a(q)=1-\left(\left(1-q+X^{*}\right) q^{m-2}\right) /\left(p^{*} p_{\bar{d}}\right)$ and $b(q)=-\left(q^{m-2} / p_{\bar{d}}\right)\left(X^{*}-X_{\bar{d}}\right)$. This can be solved as

$$
p^{*}(q)-p_{\bar{d}}(q)=e^{A(q)}\left(p^{*}\left(q_{1}\right)-p_{\bar{d}}\left(q_{1}\right)+\int_{q_{1}}^{q} e^{-A\left(q^{\prime}\right)} b\left(q^{\prime}\right) d q^{\prime}\right),
$$

where $A(q)=-\int_{1 / 2}^{q} a\left(q^{\prime}\right) / q^{\prime} d q^{\prime} . A(q)$ is evaluated as follows. By using (29) and (30), we see that

$$
\begin{aligned}
& X_{\bar{d}}(q)=-f^{c^{*}}\left(\lambda_{+}^{\bar{d}}\left(c^{*}\right)\right)(q-1)+o(q-1), \\
& X^{*}(q)=-f^{c^{*}}\left(\lambda_{+}^{d}\left(c^{*}\right)\right)(q-1)+o(q-1), \\
& p_{\bar{d}}(q)=\lambda_{+}^{\bar{d}}\left(c^{*}\right)(q-1)+o(q-1), \\
& p^{*}(q)=\lambda_{+}^{d}\left(c^{*}\right)(q-1)+o(q-1) .
\end{aligned}
$$

These expressions easily prove that there exists sufficiently small $\epsilon>0$ such that

$$
a(q)<1-\frac{C_{1}}{1-q} \quad \text { for } \quad q \in[1-\epsilon, 1)
$$

with some positive constant $C_{1}$. Hence, we have

$$
-\frac{a(q)}{q}>\frac{C_{1}-(1-q)}{(1-q) q}>\frac{C_{1}-\epsilon}{1-q}>0
$$

for $q \in[1-\epsilon, 1)$, so that

$$
\begin{aligned}
A(q) & =-\int_{1 / 2}^{q} \frac{a\left(q^{\prime}\right)}{q^{\prime}} d q^{\prime}=-\int_{1 / 2}^{1-\epsilon} \frac{a\left(q^{\prime}\right)}{q^{\prime}} d q^{\prime}-\int_{1-\epsilon}^{q} \frac{a\left(q^{\prime}\right)}{q^{\prime}} d q^{\prime} \\
& >A(1-\epsilon)+\int_{1-\epsilon}^{q} \frac{C_{1}-\epsilon}{1-q^{\prime}} d q^{\prime}=A(1-\epsilon)+\left(C_{1}-\epsilon\right) \log \left(\frac{\epsilon}{1-q}\right) .
\end{aligned}
$$

Thus, we have obtained the required inequality

$$
e^{A(q)}>e^{A(1-\epsilon)} e^{\log (\epsilon /(1-q))^{C_{1}-\epsilon}}=e^{A(1-\epsilon)}\left(\frac{\epsilon}{1-q}\right)^{C_{1}-\epsilon} .
$$

Now, take the limit $q \rightarrow 1$ in (31). Noting that (29) and (32), we obtain the relation

$$
p^{*}\left(q_{1}\right)-p_{\bar{d}}\left(q_{1}\right)+\int_{q_{1}}^{1} e^{-A\left(q^{\prime}\right)} b\left(q^{\prime}\right) d q^{\prime}=0 .
$$


Then, take the limit $q_{1} \rightarrow 0$ in the above relation. By (29), we finally conclude that

$$
\int_{0}^{1} e^{-A\left(q^{\prime}\right)} b\left(q^{\prime}\right) d q^{\prime}=0,
$$

which is impossible because $b(q)>0$ for $0<q<1$. This proves that $\mathcal{U}_{\bar{d}}\left(c^{*}\right)$ starting from $P_{1}$ cannot approach $P_{c^{*}}$ as $z \rightarrow \infty$. Therefore, it must approach $P_{0}$ as $z \rightarrow \infty$. This completes the proof.

Lemmas 11 and 13 assure that the existence of a unique connection orbit of (15) only for each $c \geq c_{d}^{*}$, that is, $c_{d}^{*}$ is the minimal wave speed. Furthermore, with the aid of Lemma 14 we can show that $c_{\bar{d}}^{*}<c_{d}^{*}$ for $\bar{d}>d$. In fact, Lemma 14 asserts that for $c=c_{d}^{*}$ there exists an orbit connecting $P_{1}$ with $P_{0}$ for $\bar{d}>d$, so that $c_{\bar{d}}^{*} \leq c_{d}^{*}$ if $\bar{d}>d$. If $c_{d}^{*}=c_{\bar{d}}^{*}$, the definition of $c_{\bar{d}}^{*}$ implies that $\mathcal{U}_{\bar{d}}\left(c_{\bar{d}}^{*}\right)$ connects $P_{1}$ with $P_{c_{\bar{d}}^{*}}$. This is a contradiction. Combining these results, we obtain the following theorem.

Theorem 15. Assume that $0<d<1$. Then, there exists some $c_{d}^{*}$, such that a traveling front solution for (10) exists uniquely (except translation) only for each $c \geq c_{d}^{*}$. Furthermore, the minimal wave speed $c_{d}^{*}$ is strictly monotone decreasing with respect to $d$, and it satisfies that $c_{1}^{*}<c_{d}^{*}<c_{0}^{*}$.

Proof. It suffices for us to prove that $c_{1}^{*}<c_{d}^{*}$. For $d=1$, the system (15) becomes

$$
\left\{\begin{array}{l}
X^{\prime}=-\frac{c}{d} X \\
q^{\prime}=p q \\
p^{\prime}=-p(p+c)-(1-q+X) q^{m-1}
\end{array}\right.
$$

The first equation of (33) with the boundary condition (13) gives that $X \equiv 0$. Therefore, we consider the problem in the invariant manifold $X=0$ in $\operatorname{cl}\left(\Omega^{+}\right)$, which is denoted by $\Omega_{0}=\{(X, q, p): X=0,0<q<1, p<0\}$. In $\Omega_{0}$, (33) is reduced to

$$
\left\{\begin{array}{l}
q^{\prime}=p q \\
p^{\prime}=-p(p+c)-(1-q) q^{m-1}
\end{array}\right.
$$

The system (34) has three critical points $P_{0}^{0}=(0,0), P_{1}^{0}=(1,0)$ and $P_{c}^{0}=(0,-c)$ in the $(q, p)$-space. At $P_{1}^{0}=(1,0)$, the eigenvalues are $\lambda_{1}^{1}(c)<0$ and $\lambda_{+}^{1}(c)>0$, and hence $P_{1}^{0}$ has the 1 -dim unstable manifold $\mathcal{U}_{1}(c)$. The slope of $\mathcal{U}_{1}(c)$ at $q=1$ is $\lambda_{+}^{1}(c)$.

Now, consider the region $\Omega_{1}^{*}=\left\{(q, p): 0<q<1, p^{*}(q)<p<0\right\}$, where $\left(X^{*}(q), q, p^{*}(q)\right)$ is the same as before. We should note that $p^{*}(1)=0, p^{*}(0)=-c_{d}^{*}$ and $p^{* \prime}(0)=\lambda_{+}^{d}\left(c_{d}^{*}\right)$. The boundary of $\Omega_{1}^{*}$ consists of $\mathcal{C}=\{(q, p): 0<q<1, p=$ $\left.p^{*}(q)\right\}, l_{1}=\{(q, p): 0<q<1, p=0\}, l_{2}=\left\{(q, p): q=0,-c_{d}^{*}<p<0\right\}, P_{0} P_{1}$ and $P_{c}$. Let us examine the behavior of the orbit $\mathcal{U}_{1}\left(c_{d}^{*}\right)$. Since $\lambda_{+}^{1}\left(c_{d}^{*}\right)<\lambda_{+}^{d}\left(c_{d}^{*}\right)$ by $(19), \mathcal{U}_{1}\left(c_{d}^{*}\right)$ enters $\Omega_{1}^{*}$. The argument in the proof of Lemma 14 with $X=0$ 


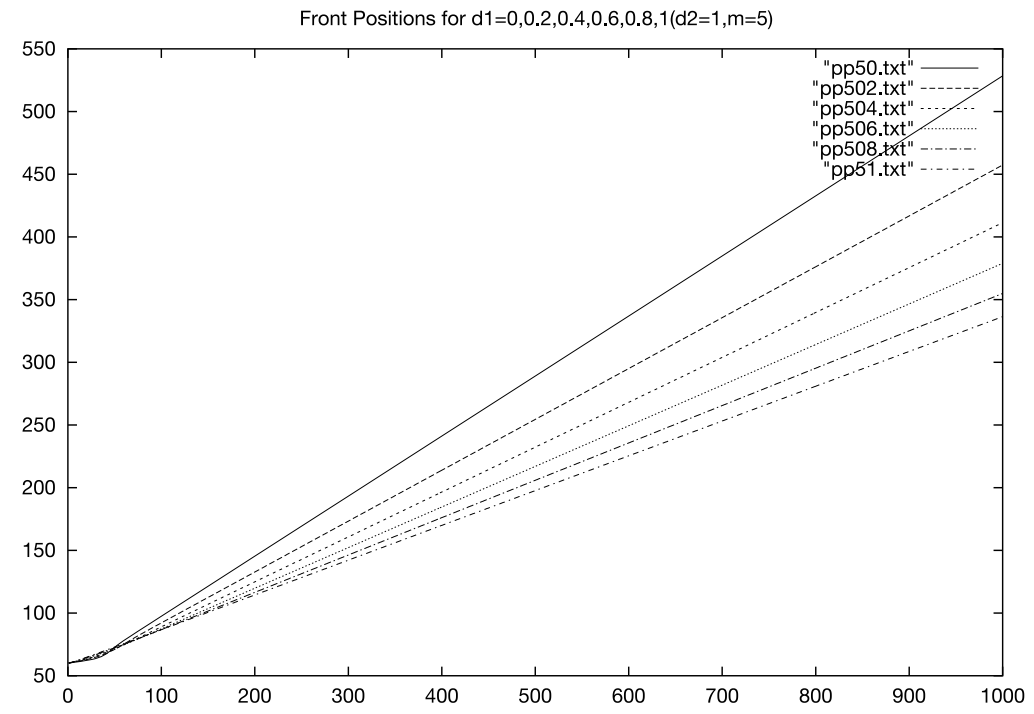

Fig. 1. The position of the front $x(t)$ of $u(x(t), t)=1 / 2(0 \leq t \leq 1000)$ for $d=0$ (pp50), 0.2 (pp502), 0.4 (pp504), 0.6 (pp506), 0.8 (pp508), 1.0 (pp51), $m=5$.

verifies that the inner products of the vector field (34) with $c=c_{d}^{*}$ and the outward normal on $\mathcal{C}, l_{1}$ and $l_{2}$ are all negative, so that that $\mathcal{U}_{1}\left(c_{d}^{*}\right)$ cannot leave $\Omega_{1}^{*}$ for all $z$. This assures the existence of the connection orbit of (34) approaching $P_{0}$ or $P_{c}$ for $c=c_{d}^{*}$, which implies that $c_{1}^{*} \leq c_{d}^{*}$ for any fixed $d \in(0,1)$. If $c_{1}^{*}=c_{d}^{*}$, choose $d^{\prime}$ satisfying $d<d^{\prime}<1$. Then $c_{1}^{*} \leq c_{d^{\prime}}^{*}$ and $c_{d^{\prime}}^{*}<c_{d}^{*}$. This is a contradiction. Hence we have $c_{1}^{*}<c_{d}^{*}$, which completes the proof.

Fig. 1 shows the numerical result of the propagation speeds of the traveling fronts obtained by solving the evolutional system (10) with the appropriate initial data of the step function type. This result illustrates numerically the last assertion of Theorem 15.

\section{The case $d_{1}>d_{2}>0$}

The condition $d_{1}>d_{2}>0$ implies $d=d_{1} / d_{2}>1$. Then, it follows from Proposition 1 that $X=U+V-1<0$. Hence, for $d>1$, our problem is to find an orbit of (15) connecting $P_{1}=(0,1,0)$ with $P_{0}=(0,0,0)$ or with $P_{c}=(0,0,-c)$, which lies entirely in $\Omega^{-}=\{(X, q, p): X<0,0<q<1, p<0\}$. Repeating the similar arguments in Section 3, we can prove the existence of the connection orbits. Therefore, we discuss only the different points from the case that $0<d<1$.

In order to examine the local behavior of the unstable manifold $\mathcal{U}_{d}(c)$ near $P_{1}$, it suffices to refer to the statements for $d>1$ in Propositions 7 and 8 . They assert that $\mathcal{U}_{d}(c)$ has the tangential direction $\mathbf{p}_{+}^{d}(c)=\left(-f^{c}\left(\lambda_{+}^{d}(c)\right), 1, \lambda_{+}^{d}(c)\right)$ at $P_{1}$. Since $f^{c}\left(\lambda_{+}^{d}(c)\right)$ is negative, $\mathcal{U}_{d}(c)$ enters $\Omega^{-}$. 
To discuss the global behavior of $\mathcal{U}_{d}(c)$, we require the connection orbit of (15) for $d=1$, which was already considered in the proof of Theorem 15 . For $d=1$, we have the system (34), and we know that there exists a unique orbit connecting $P_{1}^{0}$ with $P_{0}^{0}$ for $c>c_{1}^{*}$ and with $P_{c}^{0}$ for $c=c_{1}^{*}$, which was assured in the proof of Theorem 2. Now, we denote this unique orbit by $\left(q, \phi_{c}(q)\right)$ for $c \geq c_{1}^{*}$, which satisfies

$$
\frac{d}{d q} \phi_{c}=-\frac{\phi_{c}\left(\phi_{c}+c\right)+(1-q) q^{m-1}}{\phi_{c} q} .
$$

Let us define the region $\Omega_{1}^{-}$by

$$
\Omega_{1}^{-} \equiv\left\{(X, q, p): q-1<X<0,0<q<1, \phi_{c}(q)<p<0\right\} .
$$

Then, the boundary $\partial \Omega_{1}^{-}$of $\Omega_{1}^{-}$consists of the followings:

$$
\begin{aligned}
& S_{1}^{-}=\left\{(X, q, p): 0<q<1, X=q-1, \phi_{c}(q)<p<0\right\}, \\
& S_{2}^{-}=\left\{(X, q, p): 0<q<1, q-1<X<0, p=\phi_{c}(q)\right\}, \\
& S_{3}^{-}=\left\{(X, q, p): 0<q<1, X=0, \phi_{c}(q)<p<0\right\}, \\
& S_{4}^{-}=\{(X, q, p): 0<q<1, q-1<X<0, p=0\}, \\
& S_{5}^{-}=\left\{(X, q, p): q=0,-1 \leq X<0, p_{1}(0) \leq p \leq 0\right\}, \\
& J_{1}^{-}=\{(X, q, p): 0<q<1, X=0, p=0\}, \\
& J_{2}^{-}=\left\{(X, q, p): 0<q<1, X=0, p=\phi_{c}(q)\right\}, \\
& J_{3}^{-}=\{(X, q, p): 0<q<1, X=q-1, p=0\}, \\
& J_{4}^{-}=\left\{(X, q, p): 0<q<1, X=q-1, p=\phi_{c}(q)\right\}, \\
& I_{0}^{-}=\left\{(X, q, p): X=0, q=0, \phi_{1}(0)<p<0\right\}, \\
& P_{0}, P_{1} \text { and } P_{c} .
\end{aligned}
$$

That is,

$$
\partial \Omega_{1}=\left(\bigcup_{i=1}^{5} S_{i}^{-}\right) \cup\left(\bigcup_{i=1}^{4} J_{i}^{-}\right) \cup I_{0}^{-} \cup P_{0} \cup P_{1} \cup P_{c} .
$$

Here, note that $J_{0}=\varnothing$ for any $c>c_{0}^{*}$. We have the followings which correspond to Proposition 6 and Lemma 9 respectively.

Proposition 16. Let $d>1$ and $c \geq c_{1}^{*}$. Any orbit of (15) starting from a point $\mathbf{u}_{0} \in \Omega_{1}^{-}$, denoted by $\mathbf{u}\left(z ; \mathbf{u}_{0}\right)$, stays in $\Omega_{1}^{-}$for all $z \geq 0$.

Proof. We only consider $S_{1}^{-}$and $S_{5}^{-} . S_{5}^{-}$is an invariant manifold, so that $\mathbf{u}\left(z ; \mathbf{u}_{0}\right)$ cannot reach any point of this surface. On $S_{1}^{-}$, the outward normal $\mathbf{n}_{1}^{-}$is $(-1,1,0)$, so that the inner product

$$
\mathbf{n}_{1}^{-} \cdot \mathbf{f}_{d}=\frac{c}{d} X+\left(\frac{1}{d}-1\right) p q+p q=\frac{c}{d}(q-1)+\frac{1}{d} p q<0 .
$$


Hence, $\mathbf{u}\left(z ; \mathbf{u}_{0}\right)$ cannot leave $\Omega_{1}^{-}$through $S_{1}^{-}$. To prove the remaining part, it suffices for us to follow the argument in the proof of Proposition 6 by replacing $\psi_{c}$ with $\phi_{c}$. This completes the proof.

Lemma 17. Assume that $d>1$. Then, for each $c \geq c_{1}^{*}$, there exists an orbit of (15) which connects $P_{1}$ with $P_{0}$.

Proof. Since $0<\lambda_{+}^{d}<\lambda_{+}^{1}$ and $-1=f^{c}(0)<f^{c}\left(\lambda_{+}^{d}\right)<f^{c}\left(\lambda_{+}^{1}\right)=0, \mathcal{U}_{d}(c)$ enters $\Omega_{1}^{-}$. Then Proposition 16 assures that $q \rightarrow 0$ as $z \rightarrow \infty$, since $q^{\prime}=p q<0$ in $\Omega_{1}^{-}$. The first equation of (15) gives (23) with $c$ in place of $c^{*}$, so that we have

$$
\begin{aligned}
|X(z)| & \leq e^{-(c / d)\left(z-z_{1}\right)}\left(\left|X\left(z_{1}\right)\right|+\int_{z_{1}}^{z} e^{(c / d)\left(\zeta-z_{1}\right)}|p(\zeta)| q(\zeta) d \zeta\right) \\
& \leq e^{-(c / d)\left(z-z_{1}\right)}\left|X\left(z_{1}\right)\right|+\frac{d}{c} M_{p} q\left(z_{1}\right),
\end{aligned}
$$

for any $z, z_{1}$ satisfying $z>z_{1}$, where $M_{p}=\max _{0 \leq q \leq 1}\left|\phi_{c}(q)\right|$. Taking the limit $z \rightarrow \infty$ in the above inequality, we have $\lim _{z \rightarrow \infty}|X(z)| \leq(d / c) M_{p} q\left(z_{1}\right)$. Again take the limit $z_{1} \rightarrow \infty$. Then we see $\lim _{z \rightarrow \infty}|X(z)|=0$. This implies that $\mathcal{U}_{d}(c)$ must approach $\mathrm{cl}\left(I_{0}^{-}\right)$, which assures that $\mathcal{U}_{d}(c)$ approach $P_{0}$ or $P_{c}$ as $z \rightarrow \infty$. To show that $\mathcal{U}_{d}(c)$ cannot approach to $P_{c}$ as $z \rightarrow \infty$ for any $c \geq c_{1}^{*}$, it suffices for us to repeat the corresponding part of the proof of Lemma 9. This completes the proof.

We define $W_{c}^{-}$by

$$
W_{c}^{-}=\left\{\mathbf{u}=(X, q, p): q-1 \leq X \leq 0, l_{c}(p) \leq q \leq 1,-c \leq p \leq 0\right\} .
$$

This plays the same role as $W_{c}$ in the Subsection 3.3, and we can construct the Wazewski set $\tilde{W}^{-}=\left\{\mathbf{U}=(\mathbf{u}, c): \mathbf{u} \in W_{c}^{-}, c_{1} \leq c \leq c_{2}\right\}$ for the extended system (22). By noting that $X<0$ and $1-q+X>0$, the whole arguments in the Subsection 3.3 with $c_{1}^{*}$ in place of $c_{0}^{*}$ are valid for $d>1$. Thus we have

Lemma 18. Assume that $d>1$. Then, there exists some positive constant $c_{d}^{*}$ $\left(<c_{1}^{*}\right)$ such that, for $c=c_{d}^{*}$, (15) has an orbit connecting $P_{1}$ with $P_{c}$.

REMARK 3. Remark 1 is also valid for $d>1$.

In order to prove that $c_{d}^{*}$ is the minimal wave speed, we introduce the region $\Omega_{-}^{*}$ by $\Omega^{*}$ with the reversed inequality with respect to $X$, that is,

$$
\Omega_{-}^{*} \equiv\left\{(X, q, p): X^{*}(q)<X<0,0<q<1, p^{*}(q)<p<0\right\},
$$

where $\left(X^{*}(q), q, p^{*}(q)\right)$ denotes the orbit connecting $P_{1}$ with $P_{c}$ for $c=c_{d}^{*}$. Then, the boundary of $\Omega_{-}^{*}$ is given by

$$
\partial \Omega_{-}^{*}=\left(\bigcup_{i=1}^{4} S_{i}^{-*}\right) \cup\left(\bigcup_{i=1}^{4} J_{i}^{-*}\right) \cup I_{0}^{-*} \cup P_{0} \cup P_{1} \cup P_{c_{d}^{*}}
$$




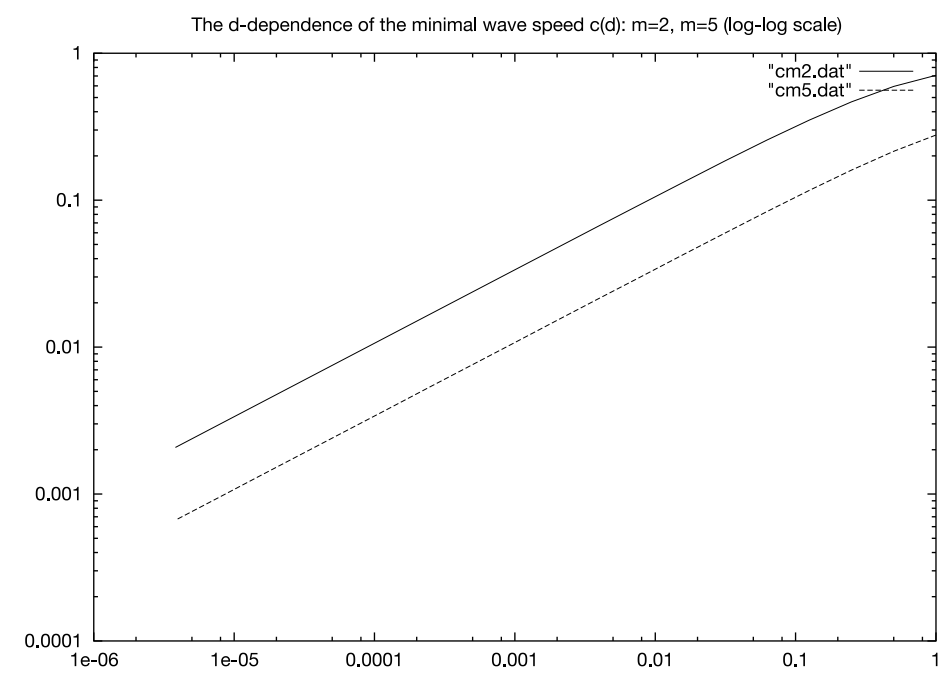

Fig. 2. The dependency of $c_{d}^{*}$ on $\delta=1 / d$ (log-log scale).

where $S_{i}^{-*}, J_{i}^{-*}(i=1,2,3,4)$ and $I_{0}^{-*}$ are $S_{i}^{-}, J_{i}^{-}(i=1,2,3,4)$ and $I_{0}^{-}$with $p^{*}(q)$ in place of $\phi_{c}(q)$. Proposition 8 assures that $\mathcal{U}_{d}(c)$ enters $\Omega_{-}^{*}$ for any $c>c_{d}^{*}$, and then applying the arguments of the proofs of Proposition 12 and Lemma 13 to $\Omega_{-}^{*}$, we see

Lemma 19. Let $d>1$ be fixed. Then, for each $c>c_{d}^{*}$, there exists an orbit of (15) connecting $P_{1}$ with $P_{0}$ lying in $\Omega_{-}^{*}$. For each positive $c<c_{d}^{*}$, there exists no orbit of $(15)$ connecting $P_{1}$ with $P_{0}$ or $P_{c}$.

As for the $d$-dependence, unfortunately, Proposition 7 asserts that $\mathcal{U}_{\bar{d}}(c)$ does not enter $\Omega_{-}^{*}$ for any $\bar{d}>d>1$. Therefore, except the monotone decreasing property of $c_{d}^{*}$ with respect to $d$, we have the similar result of Theorem 15 for $d>1$.

THEOREM 20. Assume that $d>1$. Then, there exists some $c_{d}^{*}$, such that a traveling front solution for (10) exists uniquely (except translation) for each $c \geq c_{d}^{*}$. Furthermore, the minimal wave speed $c_{d}^{*}$ satisfies that $c_{d}^{*}<c_{1}^{*} \leq \sqrt{2 /(m(m-1))}$.

\section{Concluding remarks}

For all $d_{1} \geq 0$ and $d_{2}>0$, we have shown the existence of the minimal wave speed $c_{d}^{*}>0$ such that the traveling front solutions exist for any $c \geq c_{d}^{*}$.

As for the front profile, we have seen that the solutions of (5) with the minimal wave speed $c_{d}^{*}$ decay to $(U, V)=(1,0)$ exponentially as $z \rightarrow \infty$. We can also see that the the solutions of $(5)$ with the speed $c>c_{d}^{*}$ decay to $(U, V)=(1,0)$ algebraically as $z \rightarrow \infty$. This can be proved by the routine work of the local analysis of the (15) near $P_{0}$ with the aid of the center manifold theory (see, for example [12]). Of course, it is obvious that any traveling front solutions decay to $(U, V)=(0,1)$ exponentially as $z \rightarrow-\infty$. 


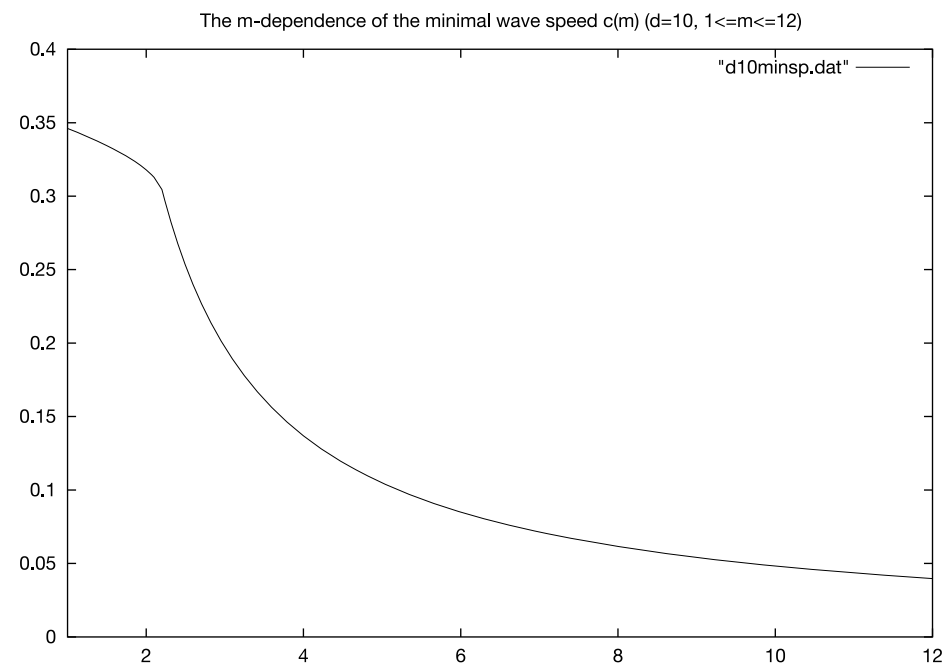

Fig. 3. The dependency of $c_{d}^{*}$ on $m(d=10,1 \leq m \leq 12)$.

We have also discussed the dependence of $c_{d}^{*}$ on the diffusion coefficients and the order of autocatalytic reactions. When $d>1$, unfortunately we cannot establish the monotone dependence of the minimal wave speed on $d$. However, Fig. 2 shows that the minimal wave speed $c_{d}^{*}$ is monotone decreasing with respect to the parameter $d$ for $m=2$ (cm2.dat) and $m=5$ (cm5.dat). This is obtained numerically by the shooting method which follows the unstable manifold of the critical point $P_{1}$ of (15). Also, this numerical result shown in Fig. 2 suggests that $c_{d}^{*}=\sqrt{\delta}\{\sigma(m)+o(1)\}$, where $\delta=1 / d$. Fig. 3 shows that he minimal wave speed $c_{d}^{*}$ is monotone decreasing with respect to the parameter $m$ for $d=10$. The justifications of these numerical results require the further study.

\section{Appendix}

We give the proof of Theorem 4 . For $d_{1}=1$ and $d_{2}=0$, (5) becomes

$$
\left\{\begin{array}{l}
U^{\prime \prime}+c U^{\prime}-U V^{m}=0 \\
c V^{\prime}+U V^{m}=0
\end{array}\right.
$$

Adding these two equations and integrating the result with the aid of (6), we have

$$
U^{\prime}+c(U+V-1)=0
$$

so that (36) is reduced to the first order system

$$
\left\{\begin{array}{l}
U^{\prime}=-c(U+V-1), \\
V^{\prime}=-\frac{1}{c} U V^{m}
\end{array}\right.
$$


which has two critical points $P_{+}=(1,0)$ and $P_{-}=(0,1)$. From Proposition 1 , we see that $U+V<1$ and $U^{\prime}>0$. This shows $c>0$. Hence we look for an orbit connecting $P_{+}$and $P_{-}$which lies entirely in $\Omega_{0}=\{(U, V): 0<U<1,0<V<$ $1, U+V<1\}$ for $c>0$.

The eigenvalues of the linearized equation of (37) about the critical point $P_{-}$ are $\lambda_{ \pm}=(1 / 2)\left[-c \pm \sqrt{c^{2}+4}\right]$. The corresponding eigenvectors are ${ }^{t}\left(c \lambda_{ \pm},-1\right)$, respectvely. Since $c \lambda_{+}=(c / 2)\left(\sqrt{c^{2}+4}-c\right)=2 /\left(\sqrt{1+4 / c^{2}}+1\right)<1$, the 1-dim unstable manifold $\mathcal{U}_{0}$ through $P_{-}$enters $\Omega_{0}$. We easily see that this $\mathcal{U}_{0}$ must stay in $\Omega_{0}$ for all $z \in \mathbb{R}$. In fact, on the boundary $l_{1}=\{(0, V): 0<V<1\}$, the vector field of $(37)$ is $(c(1-V), 0)$, and it is $\left(0, U(1-U)^{m}\right)$ on $l_{2}=\{(U, V): 0<U<$ $1, U+V=1\}$. These imply that the vector fields on $l_{1}$ and $l_{2}$ are directed to the inside of $\Omega_{0}$. The boundary $l_{3}=\{(U, 0): 0 \leq U \leq 1\}$ is an invariant manifold. Thus we know that $\mathcal{U}_{0}$ cannot traverse the boundary of $\Omega_{0}$ and stays in $\Omega_{0}$. Noting that there is no critical point in $\Omega_{0}$ and $U^{\prime}>0$, we can conclude that $\mathcal{U}_{0}$ must approach $P_{+}$as $z \rightarrow+\infty$. This completes the proof.

\section{References}

[ 1 ] J. Billingham, Phase plane analysis of one-dimensional reaction diffusion waves with degenerate reaction terms. Dynamics and Stability of Systems, 15 (2000), 23-33.

[ 2 ] J. Billingham and D.J. Needham, A note on the properties of a family of travelling-wave solutions arising in cubic autocatalysis. Dynamics and Stability of Systems, 6 (1991), 33-49.

[ 3 ] J. Billingham and D.J. Needham, The development of travelling waves in quadratic and cubic autocatalysis with unequal diffusion rates. I. Permanent form travelling waves. Phil. Trans. R. Soc. Lond. A, 334 (1991), 1-24.

[ 4 ] J. Billingham and D.J. Needham, The development of travelling waves in quadratic and cubic autocatalysis with unequal diffusion rates. II. An initial-value problem with an immobilized of nearly immobilized autocatalyst. Phil. Trans. R. Soc. Lond. A, 336 (1991), 497-539.

[ 5 ] J. Billingham and D.J. Needham, The development of travelling waves in quadratic and cubic autocatalysis with unequal diffusion rates. III. Large time development in quadratic autocatalysis. Quart. Appl. Math., 50 (1992), 343-372.

[ 6 ] S.R. Dunbar, Travelling wave solutions of diffusive Lotka-Volterra equations. J. Math. Biology, 17 (1983), 11-32.

[ 7 ] R.J. Field and M. Burger (eds.), Oscillations and Traveling Waves in Chemical Systems. Wiley, New York, 1985.

[ 8 ] S. Focant and Th. Gallay, Existence and stability of propagation fronts for an autocatalytic reaction-diffusion system. Physica D, 120 (1998), 346-368.

[ 9 ] V. Gionvangigli, Nonadiabatic plane laminar flames and their singular limits. SIAM J. Math. Anal., 21 (1990), 1305-1325.

[10] P. Gray, T.H. Merkin, D.J. Needham and S.K. Scott, The development of travelling waves in a simple isothermal chemical system. III. Cubic and mixed autocatalysis. Proc. R. Soc. Lond. A, 430 (1990), 509-524.

[11] P. Gray, S.K. Scott and K. Showalter, The influence of the form of autocatalysis on the speed of chemical waves. Phil. Trans. R. Soc. Lond. A, 337 (1991), 249-260

[12] J. Hale and H. Koçak, Dynamics and Bifurcations. Springer-Verlag, New York, 1991.

[13] Y. Hosono, Phase plane analysis of travelling waves for higher order autocatalytic reactiondiffusion systems, to appear in Discrete Contin. Dyn. Syst. Ser. B (2007).

[14] Y. Hosono and H. Kawahara, The minimal propagation speed of travelling waves for autocatalytic reaction-diffusion equations, Japan J. Industrial and Applied Math., 18 (2001), 445-458.

[15] R. Kapral and K. Showalter (eds.), Chemical Waves and Patterns. Kluwer Academic Publishers, Dordrecht, 1995. 
[16] J.H. Merkin and D.J. Needham, Propagating reaction diffusion waves in a simple isothermal quadratic autocatalytic chemical system. J. Engng. Math., 23 (1989), 343-356.

[17] J.H. Merkin and D.J. Needham Reaction-Diffusion waves in an isothermal chemical system with general orders of autocatalysis and spatial dimension. Z. angew. Mech. Phys., 44 (1993), $707-721$.

[18] M.J. Metcalf, J.H. Merkin and S.K. Scott, Oscillating wave fronts in isothermal chemical systems with arbitrary powers of autocatalysis. Proc. R. Soc. Lond. B, 447 (1994), 155-174.

[19] D.J. Needham and J.H. Merkin, The development of travelling waves in a simple isothermal chemical system with general orders of autocatalysis and decay. Phil. Trans. R. Soc. Lond. A, 337 (1991), 261-274.

[20] G. Nicolis and I. Prigogine, Self-Organization in Nonequilibrium Systems, Wiley, New York, (1977).

[21] A. de Pablo and J.L. Vazquez, Travelling wave behaviour for a Porous-Fisher equation. Euro. J. Applied Mathematics, 9 (1998), 285-304.

[22] H. Takase and B.D. Sleeman, Travelling-wave solutions to monostable reaction-diffusion systems of mixed monotone type. Proc. R. Soc. Lond. A, 455(1999), 1561-1598.

[23] A.I. Volpert, Vitaly A. Volpert and Vladimir A. Volpert, Travelling wave solution of parabolic systems. American Mathematical Society, Rhode Island, 1994. 